\title{
THICKNESS EFFECTS ON THE STABILITY OF THIN WALLED STRUCTURES
}

\author{
N. Triantafyllidis and Y. J. KwON \\ Department of Aerospace Engineering, The University of Michigan, \\ Ann Arbor, MI 48109-2140, U.S.A.
}

(Received 9 December 1986)

\begin{abstract}
A CONSISTENT method for the analysis of bifurcation instabilities in shells of arbitrary thickness has been proposed. The advantages of this method, as compared to the classical approach followed so far, are the independence of the results on the nonlinear shell theory employed as well as the possibility of finding the dependence of the critical load and eigenmode on the shell's thickness up to any degree of accuracy desired.

A brief description of the general method, which is asymptotic with respect to the shell's thickness, is followed by its application to the case of an internally or externally pressurized infinite cylindrical tube for which an analytical solution of the resulting asymptotic problem is possible. Critical loads and modes compare very favorably with a numerical solution of the exact three dimensional problem even for relatively thick tubes. The presentation is concluded with a short discussion on the application of this technique to the general wrinkling instability problem in metal forming.
\end{abstract}

\section{INTRODUCTION}

BUCKuING Is a well known instability associated with the loading of thin walled structures. The term "buckling" here is a generic one and incorporates all abrupt changes in the deformation pattern of a shell, occurring in the course of a loading process. In mathematical terms the corresponding phenomenon is called "bifurcation" and it involves the loss of uniqueness in the solution of the (always nonlinear) governing equations for the pertaining boundary value problem describing the deformation of the structure in question. Although the first buckling studies in solids go back to Euler, the proper mathematical foundation for the theory of structural stability as a bifurcation problem is a much more recent achievement and it is essentially due to the works of KOITER $(1945)$ for the elastic case and HILL $(1957,1958)$ for the more general case of rate independent solids. Consequently, the terms bifurcation and buckling are considered completely equivalent in the rest of this work.

Using the general method introduced by KorTER (1945), in the vast majority of shell stability analyses up to date, the following procedure is invariably employed: first a nonlinear shell theory is adopted which is subsequently linearized about the critical load thus yielding a (nonlinear) eigenvalue problem whose solution gives the buckling load and the corresponding eigenmode. The shortcoming of this approach is the theoretical possibility of an infinite variety of critical loads and eigenmodes 
for the same physical problem, due to the great wealth of nonlinear shell theory approximations existing in the literature. Although for shells with instabilities in the small strain elastic region of their constitutive response, the difference in the buckling results due to the choice of different nonlinear shell theories is negligible (as calculations presented subsequently in this paper will indicate), one does expect significant effects of the choice of nonlinear shell theory approximation in results involving bifurcations at rather high deformations and strains in the solid, as for example in problems associated with sheet metal forming applications (e.g. puckering, wrinkling, etc.).

It is the purpose of the present investigation to propose a consistent alternative method for the calculation of critical loads and eigenmodes in a shell bifurcation problem in a unique way, once the shell geometry and material constitutive equations have been determined. The proposed method will also provide the critical loads and eigenmodes as functions of the shell's thickness to any desired degree of accuracy, information which is impossible to obtain using the aforementioned nonlinear shell theory-dependent approach.

In place of the classical approach, in which a two dimensional nonlinear shell theory (derived from the three dimensional governing equations of the solid) is linearized about the critical load, the present method starts from the bifurcation equations of the three dimensional shell-like solid (which have been obtained by linearization about the critical load of the same three dimensional governing equations for the nonlinear solid in question) and subsequently takes the limit as the shell thickness $h$ tends to zero, following a multiple scale asymptotic technique in the spirit of the approach introduced by DESTUYNDER (1980) for the derivation of linearly elastic shell theories. A graphic presentation of both procedures (classical and proposed) is depicted in Table 1 .

In view of the considerable algebraic complexity of the theory for the case of an arbitrary shell, only a brief exposition of the general method is given. The presentation proceeds with the application of the theory to the buckling instability of an infinitely long cylindrical tube under internal and external pressure, a problem for which a general analytical three dimensional solution does not exist. An asymptotic (in terms of $\varepsilon=\ln$ (outer radius/inner radius)) analytical solution for the critical load and eigenmodes is presented for a hyperelastic as well as for an elastoplastic material. Results are compared with some recent numerical investigations on the subject due to Haughton and OGDFN (1979) and CHU (1979) for the internally pressurized elastic and elastoplastic cases respectively and TIMOSHENKo and GERE (1961) for the (classical) externally pressurized linearly elastic cylinder. The asymptotic method used here predicts several interesting features of these problems that have only been found numerically thus far. In addition to the analytical asymptotic calculations, finite element computations have been carried out for the cylinder of arbitrary thickness. Results indicate a remarkable agreement between the numerical and asymptotic values of the critical loads for thickness to radius of curvature ratios up to $20 \%$ even when only the first one or two terms in the pertaining asymptotic expansions are considered.

Finally, the presentation is concluded with some interesting remarks about the general formulation of the theory for an arbitrary shell and its applications to sheet metal forming problems. 
TABLE 1. Schematic representation of the classical and proposed approach for thin shell buckling problems

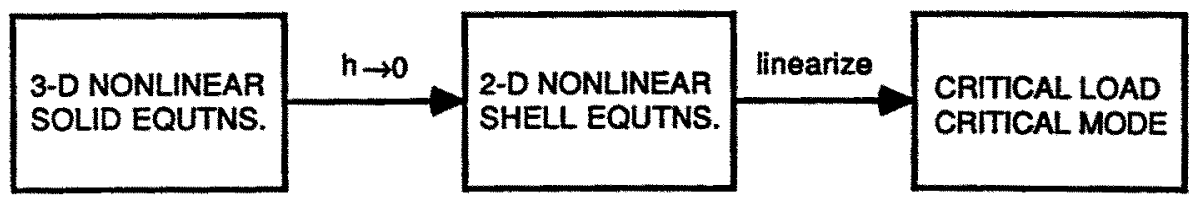

CLASSICAL APPROACH

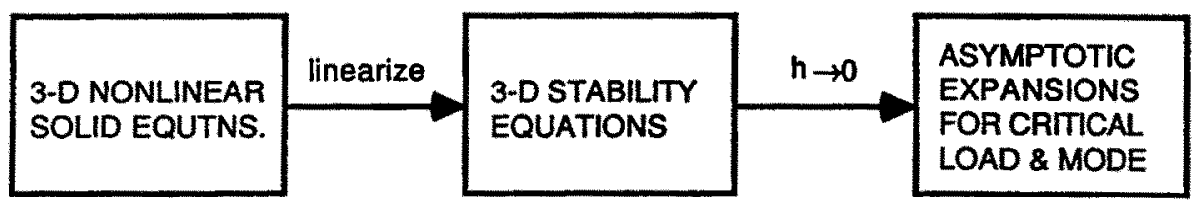

PROPOSED APPROACH

\section{PROPOSED METHOD}

As discussed in the introduction, only a brief description of the proposed method will be outlined here. For an arbitrary rate independent solid, with external loads increasing proportionally to a scalar load parameter $\lambda$, the necessary conditions for a bifurcated solution at some point $\lambda=\lambda_{c r}$ is the existence of a nontrivial solution to the homogeneous incremental equilibrium equations, which can be written as

$$
\langle\delta v, L v\rangle=0,
$$

where $L$ is a linear operator (containing the solid's incremental moduli and depending on the prebifurcation solution and hence on $\lambda$ ) operating on the bifurcation eigenmode $v$, while $\delta v$ is any arbitrary function belonging to the same function space as $v$, say $V$, with $V$ appropriately chosen in order to satisfy antomatically the essential (homogeneous) boundary conditions for the eigenmodes. In addition the $\langle\cdot, \cdot\rangle$ symbol in (2.1) denotes the duality pairing of $V$ and its dual $V^{\prime}$. The weak formulation of the bifurcation equations is to be employed in this scetion in view of its notational 
compactness. One should also mention at this point that in the case where the operator $L$ is selfadjoint, as is most often the case in nonlinear elasticity or rate independent plasticity problems, a quadratic (in $v$ ) functional $F(\lambda ; v)$ can be found whose first variation yields $(2.1)$; namely

$$
F \equiv \frac{1}{2}\langle v, L v\rangle ; \quad \delta F=0 \Leftrightarrow\langle\delta v, L v\rangle=0 .
$$

It should also be noted that a desirable feature for the bifurcation functional $F$ is that it is positive definite for values of the load parameter smaller than the lowest bifurcation load. For more details on the subject the interested reader is referred to the review articles of BUDIANSKY (1974) (for the nonlinear elasticity case) and HUTCHINSON (1974) (for the rate independent plasticity case) and the references quoted therein.

For a shell-like structure, $L$ and $v$ are functions of $x, y$ as well as $\varepsilon$ where $x$ denotes the mid-surface coordinate(s), $y$ denotes the thickness coordinate and $\varepsilon$ denotes the ratio of the shell's thickness (initial or current depending on the formulation of the problem) to another characteristic length. Although in the asymptotic analysis of linear three-dimensional shell-like structures, two small parameters do appear naturally (see DESTUYNDER, 1980), namely the thickness to characteristic mid-surface length ratio $\varepsilon$ and the thickness to minimum mid-surface radius of curvature ratio $\eta$, in the present study only one small parameter thin walled structures are to be considered. This assumption does not impair the problem's generality since in the majority of the applications considered, the reference (undeformed) mid-surface of the shell remains fixed while the corresponding initial thickness tends to zero. Hence the nonlinear (in $\lambda$ ) eigenvalue problem (2.1) (or (2.2)) has a solution of the form $\dagger$

$$
\lambda=\lambda_{c r}(\varepsilon), \quad v=v(x, y ; \varepsilon) .
$$

The proposed method consists of adopting the following transformation of variables and asymptotic expansions

$$
\begin{aligned}
v(x, y ; \varepsilon) & =\stackrel{0}{v}(x, \xi)+\varepsilon v(x, \xi)+\varepsilon^{2} v(x, \xi)+\cdots \\
\lambda_{c r}(\varepsilon) & =\hat{\lambda}_{0}+\varepsilon \hat{\lambda}_{1}+\varepsilon^{2} \lambda_{2}+\cdots ; \quad \xi \equiv y / \varepsilon
\end{aligned}
$$

which, upon substitution to (2.1) and collection of terms of like order in $\varepsilon$, yields

$$
\begin{aligned}
\left\langle\delta v, L_{0} v\right\rangle & =0 ; \quad L_{0} \equiv L\left(x, \xi ; 0, \lambda_{0}\right), \\
\left\langle\delta v, L_{0} v\right\rangle & =-\left(\left\langle\delta v,\left.\frac{\partial L}{\partial \varepsilon}\right|_{0} ^{0} \begin{array}{c}
v \\
v
\end{array}\right\rangle+\lambda_{1}\left\langle\delta v,\left.\frac{\partial L}{\partial \lambda}\right|_{0} \begin{array}{c}
v \\
v
\end{array}\right\rangle\right) ; \\
\left.\frac{\partial L}{\partial \varepsilon}\right|_{0} & =\frac{\partial L}{\partial \varepsilon}\left(x, \xi ; 0, \lambda_{0}\right),\left.\frac{\partial L}{\partial \lambda}\right|_{0}=\frac{\partial L}{\partial \lambda}\left(x, \xi ; 0, \lambda_{0}\right) .
\end{aligned}
$$

Assuming for simplicity that the eigenmode $v$ is unique, the following mode orthogonality condition is used 


$$
\left\langle\hat{v}, L_{0} v\right\rangle=\left\langle\hat{v}, \begin{array}{c}
i \\
v
\end{array} L_{0} v\right\rangle=0 \quad \text { for } i \geqslant 1
$$

which in turn can be employed for the determination of the $\lambda_{i}$ 's. Hence, from $(2.5)_{2}$ with the help of (2.6), one gets

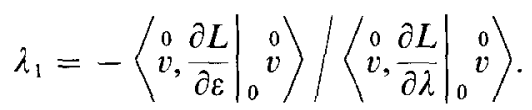

The above procedure gives in a straightforward and unique (for a given three-dimensional boundary value problem) fashion, the critical load and eigenmode of a shelllike structure, as a function of its thickness.

It can be shown (but the proof will be omitted in this work in view of the cumbersome notation needed) that the specific dependence of $L$ on $\varepsilon$ implies that the lowest order term $v$ in the eigenmode expansion depends only on the midsurface coordinates, i.e. $\stackrel{0}{v}=\stackrel{0}{v}(x)$ as is also the case in the classical approach (i.e. the buckling mode of the linearized nonlinear shell theory) although the two different approaches are expected to produce in general different results. Moreover, if the first nontrivial term in the expansion for $\lambda \dagger$ is the zeroth order one then a membrane mode prevails while if a higher order term (usually $\varepsilon^{2}$ or higher) is the first non-trivial term, the corresponding bifurcation is of the bending type. In the following sections a nontrivial problem is treated analytically and a wide range of possible types of shell bifurcations are examined using the general method proposed here.

As explained in the introduction, the particular attraction of the pressurized cylinder example treated here is the possibility of an analytical solution for the resulting asymptotic problem. Our purpose is not to provide just another solution to the classical pressurized cylinder stability problem (on which there is already a substantial amount of literature whose review is not relevant for this work) but to illustrate a novel method using this relatively simple example.

\section{Application to the Pressurization Problem of an INFINITE CYLINDER}

Attention is focussed on the influence of the wall thickness on the bifurcation behavior of an infinitely long incompressible cylinder of current (and initial) inner and outer radii $r_{i}, r_{0}$ (and $R_{i}, R_{0}$ ) respectively. In addition to its full three-dimensional character, this problem possesses a prebifurcation stress state which is not constant, (thus precluding a 3-D analytical solution), but a rather involved function of position. Both external and internal hydrostatic pressure loadings will be investigated. Two different constitutive equations will be employed; an elastoplastic power law type material and a rubber-like neo-Hookean one, in view of their entirely different behavior in the internal pressurization case. 


\section{Problem formulation}

A brief derivation of the governing equations, that fits the framework of the general formulation in Section 2, is presented here. Assume a body whose prebifurcation stress and strain distributions are known functions of the monotonically varying load parameter $\lambda$. Bifurcation occurs if, at some value of the load parameter $\lambda_{c r}$, for a given increment in load $\dot{\lambda}$ (i.e. for a given increment of the boundary prescribed loads) the solution to the rate problem for the solid ceases to be unique. In the following, an updated Lagrangian formulation of the problem will be adopted with the reference configuration taken to coincide with the current one at the onset of bifurcation. Convected curvilinear coordinates $\theta^{i}$ will be employed in the general formulation of the incremental equilibrium equations with $g^{i j}$ denoting the contravariant components of the metric tensor. An index preceded by a comma $\left.{ }_{(i}\right)$ indicates covariant differentiation with respect to the corresponding variable $\left(\theta^{i}\right)$ while a quantity surmounted by a dot $\left(^{\circ}\right)$ stands for differentiation with respect to a monotonically increasing timelike parameter (usually $\lambda$ if $\lambda$ increases or $-\lambda$ if $\lambda$ decreases during the loading process).

At bifurcation, at least two different solutions to the incremental equilibrium problem are possible and if $\Delta()$ denotes the difference of two such solutions (for a given field quantity), the incremental equilibrium equations

$$
\left(\Delta \dot{\sigma}^{i j}+\sigma^{k j} \Delta \dot{u}_{, k}^{i}\right)_{, j}=0
$$

have to be satisfied at the interior of the solid, while on the part of the surface that is subjected to a hydrostatic pressure $p$ the corresponding boundary conditions are

$$
\left(\Delta \dot{\sigma}^{i j}+\sigma^{k j} \Delta \dot{u}_{, k}^{i}\right) n_{j}=p\left(g^{i l} g^{j k}-g^{i j} g^{k l}\right) \Delta \dot{u}_{k, l} n_{j},
$$

with $n$ the outward normal to the surface in question.

For the incrementally linear, incompressible materials to be considered here, their constitutive law assumes the form

$$
\Delta \dot{\sigma}^{i j}=L^{i j k l} \Delta \dot{u}_{k, l}-\Delta \dot{\bar{p}} g^{i j}
$$

with $\bar{p}$ the hydrostatic pressure inside the solid and $\underset{\sim}{L}$ the tensor of incremental moduli. To complete the set of governing equations of bifurcation, the incompressibility condition must also be added:

$$
\Delta \dot{u}_{, k}^{k}=0
$$

It is often convenient to write incremental constitutive laws for finitely deforming solids in terms of the Jaumann (or co-rotational) derivative of stress, namely

$$
\stackrel{v}{\sigma^{i j}}=C^{i j k l} \dot{u}_{k, l}-\dot{\bar{p}} g^{i j}
$$

and in this case the new tensor of incremental moduli $\underset{\sim}{ }$ is related to the tensor $\underset{\sim}{ }$ in (3.3) by

$$
L^{i j k l}=C^{i j k l}-\frac{1}{2}\left(g^{i k} \sigma^{j l}+g^{i l} \sigma^{j k}+g^{j l} \sigma^{i k}+g^{j k} \sigma^{i l}\right) .
$$

The incremental theories to be considered are rate independent and consequently the incremental moduli $\underset{\sim}{L}$ (or $\underset{\sim}{C}$ ) depend on the current stress and deformation state and possibly on some internal variables too, but are independent of rate quantities 
(in the elastoplastic material case the total loading branch of the incremental moduli tensor will be considered).

At bifurcation, a non-trivial solution (in terms of $\Delta \dot{\underline{u}}, \Delta \dot{\bar{p}}$ ) to the linear (with respect to the aforementioned field quantities) boundary value problem (3.1)-(3.4) exists. The pointwise formulation of the general bifurcation problem has been adopted here as a starting point in view of the unboundedness of the domain of definition of the functions at hand for the infinite cylinder. For more details on the formulation of the general bifurcation problem for a rate independent solid, the interested reader is referred to the original work by HILL $(1957,1958)$ and the review articles of BUDIANSKY (1974) and HuTCHINSON (1974).

For the infinite cylinder problem investigated here, cylindrical coordinates will be used with $\stackrel{1}{\theta}=r, \stackrel{2}{\theta}=\theta, \stackrel{3}{\theta}=z$. Physical components of tensor fields will be employed with the corresponding indices included between angular brackets $(\langle\rangle)$.

Without loss of generality (more comments on this subject will be offered later), a non-axisymmetric bifurcation eigenmode is assumed to be of the form (for $n \neq 0$ )

$$
\begin{aligned}
& \Delta \dot{u}_{\langle 1\rangle}=v_{r}(r) \sin (n \theta) \sin (\omega z), \\
& \Delta \dot{u}_{\langle 2\rangle}=v_{\theta}(r) \cos (n \theta) \sin (\omega z), \\
& \Delta \dot{u}_{\langle 3\rangle}=v_{z}(r) \sin (n \theta) \cos (\omega z) .
\end{aligned}
$$

The goal will be the construction of a bifurcation functional with the properties indicated in Section 2 [see (2.2)]. For this purpose the $i$ th equilibrium equation (3.1) is multiplied by $\delta u_{i}$ (which are also assumed to have $\theta$ and $z$ dependence of the form indicaled by (3.7)) and the resulting products are summed and subsequently integrated in the intervals $r_{i} \leqslant r \leqslant r_{0}, 0 \leqslant \theta \leqslant 2 \pi, 0 \leqslant z \leqslant 2 \pi / \omega$. Upon integration by parts, employment of the boundary and incompressibility conditions (3.2), (3.4) and change of variables from $r$ to $x \equiv \ln \left(r / r_{i}\right)$, the following bifurcation functional is constructed

$$
\begin{aligned}
& I\left(\lambda ; v_{r}, v_{z}\right)=\frac{1}{2}\left\{\int _ { 0 } ^ { e } \left[\left(C_{\langle 1111\rangle}-\sigma_{\langle 11\rangle}\right)\left(v_{r, x}\right)^{2}+2 C_{\langle 1122\rangle}\left(\omega r_{i} e^{x} v_{z}\right.\right.\right. \\
& \left.-v_{r, x}\right) v_{r, x}+2 C_{\langle 1133\rangle}\left(-\omega r_{i} e^{x} v_{z}\right) v_{r, x}+\left(C_{\langle 2222\rangle}-\sigma_{\langle 22\rangle}\right)\left(\omega r_{i} e^{x} v_{z}\right. \\
& \left.-v_{r, x}\right)^{2}+2 C_{\langle 2233\rangle}\left(\omega r_{i} e^{x} v_{z}-v_{r, x}\right)\left(-\omega r_{i} e^{x} v_{z}\right)+\left(C_{\langle 3333\rangle}-\sigma_{\langle 33\rangle}\right) \\
& \quad \times\left(-\omega r_{i} e^{x} v_{z}\right)^{2}+\left(C_{\langle 1212\rangle}+\frac{\sigma_{\langle 11\rangle}-\sigma_{\langle 22\rangle}}{2}\right) \frac{1}{n^{2}}\left(v_{r, x x}+v_{r, x}-\omega r_{i} e^{x} v_{z}\right. \\
& \left.-\omega r_{i} e^{x} v_{z, x}\right)^{2}+2\left(C_{\langle 1212\rangle}-\frac{\sigma_{\langle 11\rangle}+\sigma_{\langle 22\rangle}}{2}\right) \frac{1}{n^{2}}\left(v_{r, x x}+v_{r, x}-\omega r_{i} e^{x} v_{z}\right. \\
& \left.-\omega r_{i} e^{x} v_{2, x}\right)\left(\left(n^{2}-1\right) v_{r}-v_{r, x}+\omega r_{i} e^{x} v_{z}\right)+\left(C_{\langle 1212\rangle}+\frac{\sigma_{\langle 22\rangle}-\sigma_{\langle 11\rangle}}{2}\right) \\
& \quad \times \frac{1}{n^{2}}\left(\left(n^{2}-1\right) v_{r}-v_{r, x}+\omega r_{i} e^{x} v_{z}\right)^{2}+\left(C_{\langle 1313\rangle}+\frac{\sigma_{\langle 11\rangle}-\sigma_{\langle 33\rangle}}{2}\right)\left(v_{z, x}\right)^{2}
\end{aligned}
$$




$$
\left.\begin{array}{l}
+2\left(C_{\langle 1313\rangle}-\frac{\sigma_{\langle 11\rangle}+\sigma_{\langle 33\rangle}}{2}\right)\left(\omega r_{i} e^{x} v_{r}\right) v_{z, x}+\left(C_{\langle 1313\rangle}+\frac{\sigma_{\langle 33\rangle}-\sigma_{\langle 11\rangle}}{2}\right) \\
\times\left(\omega r_{i} e^{x} v_{r}\right)^{2}+\left(C_{\langle 2323\rangle}+\frac{\sigma_{\langle 22\rangle}-\sigma_{\langle 33\rangle}}{2}\right)\left(n v_{z}\right)^{2}+2\left(C_{\langle 2323\rangle}-\frac{\sigma_{\langle 22\rangle}+\sigma_{\langle 33\rangle}}{2}\right) \\
\times\left(\omega r_{i} e^{x} v_{z}\right)\left(v_{r, x}+v_{r}-\omega r_{i} e^{x} v_{z}\right)+\left(C_{\langle 2323\rangle}+\frac{\sigma_{\langle 33\rangle}-\sigma_{\langle 22\rangle}}{2}\right)\left(\frac{\omega r_{i} e^{x}}{n}\right)^{2} \\
\left.\times\left(v_{r, x}+v_{r}-\omega r_{i} e^{x} v_{z}\right)^{2}\right] d x+\left[p \left(v_{r}^{2}+\frac{1}{n^{2}}\left(v_{r, x}+v_{r}-\omega r_{i} e^{x} v_{z}\right)^{2}\right.\right. \\
\left.\left.\quad-2 v_{r}\left(v_{r, x}+v_{r}\right)\right)\right]_{x=0}^{x=8}
\end{array}\right\},
$$

where $[f]_{a}^{b} \equiv f(b)-f(a)$ and $\varepsilon \equiv \ln \left(r_{0} / r_{i}\right)$ is the current thickness parameter of the shell. The Euler equations of the above functional are the bifurcation equations for the cylinder corresponding to the nonaxisymmetric mode (3.7) as one can verify. In the derivation of (3.8) it was tacitly assumed that in the prebifurcation state the material is orthotropic with principal directions $(r, \theta, z)$ which are also the directions of the principal stresses, conditions which hold true for all the cases to be subsequently analyzed. It should also be noted that in view of the nature of the prebifurcation solution, the incremental moduli tensor $C$ as well as the components of the stress tensor $g$ entering (3.8) are functions of the thickness variable $x$ (and the load parameter $\lambda$ of course).

Following the general method of Section 2, the thickness variable will be rescaled with the introduction of $\xi \equiv x / \varepsilon$ where the normalized thickness variable $\xi$ varies $0 \leqslant \xi \leqslant 1$. The bifurcation functional $I$ in (3.8) can be simplified further by exploiting the only nontrivial equilibrium equation of the prebifurcation condition, namely

$$
\left(\sigma_{\langle 11\rangle}\right)_{, x}=\sigma_{\langle 22\rangle}-\sigma_{\langle 11\rangle}, \quad \sigma_{\langle 11\rangle}(0)=-p_{i}, \quad \sigma_{\langle 11\rangle}(\varepsilon)=-p_{0},
$$

to yield the expression

$$
\begin{aligned}
F\left[\lambda ; v_{r}(\xi), v_{z}(\xi)\right]= & \frac{1}{2} \int_{0}^{1}\left[\bar{A}_{11}\left(v_{r, \xi \xi}\right)^{2}+2 \bar{A}_{12} v_{r, \xi \xi} v_{r, \xi}+2 \bar{A}_{13} v_{r, \xi \xi} v_{r}\right. \\
+2 \bar{A}_{14} v_{r, \xi \xi} v_{z, \xi}+ & 2 \bar{A}_{15} v_{r, \xi \xi} v_{z}+\bar{A}_{22}\left(v_{r, \xi}\right)^{2}+2 \bar{A}_{23} v_{r, \zeta} v_{r}+2 \bar{A}_{24} v_{r, \xi} v_{z, \xi}+2 \bar{A}_{25} v_{r, \xi} v_{z}+\bar{A}_{33}\left(v_{r}\right)^{2} \\
& \left.+2 \bar{A}_{34} v_{r} v_{z, \xi}+2 \bar{A}_{35} v_{r} v_{z}+\bar{A}_{44}\left(v_{z, \xi}\right)^{2}+2 \bar{A}_{45} v_{z, \xi} v_{z}+\bar{A}_{55}\left(v_{z}\right)^{2}\right] d \xi, \quad(3.10)
\end{aligned}
$$

where $F=\varepsilon^{3} I$ and the coefficients $\bar{A}_{i j} 1 \leqslant i, j \leqslant 5$ are given by

$\bar{A}_{11}=\left(c_{\langle 1212\rangle}+\frac{\sigma_{\langle 11\rangle}-\sigma_{\langle 3 \eta\rangle}}{2}\right) \frac{1}{n^{2}}$

$\bar{A}_{12}=0$ 


$$
\begin{aligned}
& \vec{A}_{13}=\varepsilon^{2}\left(C_{\langle 1212\rangle}+\frac{\sigma_{\langle 11\rangle}-\sigma_{\langle 22\rangle}}{2}\right) \frac{n^{2}-1}{n^{2}}, \\
& \bar{A}_{14}=\varepsilon\left(C_{\langle 1212\rangle}+\frac{\sigma_{\langle 11\rangle}-\sigma_{\langle 22\rangle}}{2}\right) \frac{\left(-\omega r_{t} e^{\varepsilon z}\right)}{n^{2}}, \\
& \bar{A}_{15}=0, \\
& \bar{A}_{22}=\varepsilon^{2}\left[C_{\langle 1111\rangle}-2 C_{\langle 1122\rangle}+C_{\langle 2222\rangle}+\sigma_{\langle 11\rangle}-\sigma_{\langle 22\rangle}\right.
\end{aligned}
$$

$$
\left.+\left(C_{\langle 2323\rangle}+\frac{\sigma_{\langle 33\rangle}-\sigma_{\langle 22\rangle}}{2}\right) \frac{\left(\omega r_{i} e^{\varepsilon \xi}\right)^{2}}{n^{2}}\right]
$$

$\bar{A}_{23}=\varepsilon^{3}\left(C_{\langle 2323\rangle}+\frac{\sigma_{\langle 33\rangle}-\sigma_{\langle 22\rangle}}{2}\right) \frac{\left(\omega r_{i} e^{\varepsilon \xi}\right)^{2}}{n^{2}}$

$\bar{A}_{24}=0$,

$$
\begin{aligned}
\bar{A}_{25}=\varepsilon^{3}\left[C_{\langle 1122\rangle}-C_{\langle 1133\rangle}+\right. & C_{\langle 2233\rangle}-C_{\langle 2222\rangle}+C_{\langle 2323\rangle}+\frac{\sigma_{\langle 22\rangle}-\sigma_{\langle 33\rangle}}{2} \\
& \left.-\left(C_{\langle 2323\rangle}+\frac{\sigma_{\langle 33\rangle}-\sigma_{\langle 22\rangle}}{2}\right) \frac{\left(\omega r_{i} e^{\varepsilon \xi}\right)^{2}}{n^{2}}\right]\left(\omega r_{i} e^{\varepsilon \xi}\right),
\end{aligned}
$$$$
\bar{A}_{33}=\varepsilon^{4}\left[C_{\langle 1212\rangle} \frac{\left(n^{2}-1\right)^{2}}{n^{2}}+\left(\frac{\sigma_{\langle 22\rangle}-\sigma_{\langle 11\rangle}}{2}\right) \frac{n^{4}-1}{n^{2}}+\left(C_{\langle 1313\rangle}\right.\right.
$$$$
\left.\left.+\frac{\sigma_{\langle 33\rangle}-\sigma_{\langle 11\rangle}}{2}\right)\left(\omega r_{i} e^{\varepsilon \xi}\right)^{2}+\left(C_{\langle 2323\rangle}+\frac{\sigma_{\langle 33\rangle}-\sigma_{\langle 22\rangle}}{2}\right) \frac{\left(\omega r_{i} e^{\varepsilon \xi}\right)^{2}}{n^{2}}\right]
$$$$
\bar{A}_{34}=\varepsilon^{3}\left[C_{\langle 1313\rangle}-C_{\langle 1212\rangle}+\frac{\sigma_{\langle 22\rangle}-\sigma_{\langle 33\rangle}}{2}+\left(C_{\langle 1212\rangle}+\frac{\sigma_{\langle 11\rangle}-\sigma_{\langle 22\rangle}}{2}\right) \frac{1}{n^{2}}\right]\left(\omega r_{i} e^{\varepsilon \xi}\right) \text {, }
$$$$
\bar{A}_{35}=\varepsilon^{4}\left[C_{\langle 2323\rangle}+\frac{\sigma_{\langle 22\rangle}-\sigma_{\langle 33\rangle}}{2}-\left(C_{\langle 2323\rangle}+\frac{\sigma_{\langle 33\rangle}-\sigma_{\langle 22\rangle}}{2}\right) \frac{\left(\omega r_{i} e^{E \xi}\right)^{2}}{n^{2}}\right]\left(\omega r_{i} e^{\varepsilon \xi}\right),
$$$$
\bar{A}_{44}=\varepsilon^{2}\left[C_{\langle 1313\rangle}+\frac{\sigma_{\langle 11\rangle}-\sigma_{\langle 33\rangle}}{2}+\left(C_{\langle 1212\rangle}+\frac{\sigma_{\langle 11\rangle}-\sigma_{\langle 22\rangle}}{2}\right) \frac{\left(\omega r_{i} e^{e \xi}\right)^{2}}{n^{2}}\right] \text {, }
$$

$\vec{A}_{45}=0$,

$$
\begin{array}{r}
\vec{A}_{5 s}=\varepsilon^{4}\left[\left(C_{\langle 2323\rangle}+\frac{\sigma_{\langle 22\rangle}-\sigma_{\langle 33\rangle}}{2}\right) n^{2}+\left(C_{\langle 2223\rangle}+C_{\langle 3333\rangle}-2 C_{\langle 2233\rangle}\right.\right. \\
\left.\left.-2 C_{\langle 2323\rangle}\right)\left(\omega r_{i} e^{\varepsilon \xi}\right)^{2}+\left(C_{\langle 2323\rangle}+\frac{\sigma_{\langle 33\rangle}-\sigma_{\langle 22\rangle}}{2}\right) \frac{\left(\omega r_{i} e^{\varepsilon^{\xi \zeta}}\right)^{4}}{n^{2}}\right] .
\end{array}
$$


It is understood that rigid body modes have to be excluded from $F$. In this particular case, from the requirement that the difference in two incremental strain tensors is null, i.e., $\Delta \dot{E}_{i j}=\Delta\left[\frac{1}{2}\left(\dot{u}_{i, j}+\dot{u}_{j, i}\right)\right]=0$, one deduces that the most general form of a rigid body mode for the case of a nonaxisymmetric mode of deformation is

$$
\left(\Delta \dot{u}_{\langle 1\rangle}, \Delta \dot{u}_{\langle 2\rangle}, \Delta \dot{u}_{\langle 3\rangle}\right)_{R . B .}=\left\{\begin{array}{l}
(z \sin \theta, z \cos \theta,-r \sin \theta) \\
(z \cos \theta,-z \sin \theta,-r \cos \theta) \\
(\sin \theta, \cos \theta, 0) \\
(\cos \theta,-\sin \theta, 0)
\end{array}\right.
$$

and hence to the variational equation $\delta F=0$, one has to add the essential boundary condition

$$
v_{r}(0)=0 \quad \text { for } n=1 .
$$

Obviously for the case of an axisymmetric deformation $(n=0)$, the bifurcation functional $F$ in (3.10) becomes singular and hence a reformulation of the problem is required. Starting from the assumption that the $(\theta$ independent) bifurcation eigenmode in this case is of the form

$$
\begin{aligned}
\Delta \dot{u}_{\langle 1\rangle} & =\omega r_{i} e^{-x} V(x) \sin (\omega z), \\
\Delta \dot{u}_{\langle 2\rangle} & =W(x) \sin (\omega z), \\
\Delta \dot{u}_{\langle 3\rangle} & =e^{-2 x} \frac{\mathrm{d} V}{\mathrm{~d} x} \cos (\omega z),
\end{aligned}
$$

where the eigenmodes are chosen so as to satisfy automatically the incompressibility condition (3.4), and following the same steps as in the derivation of the bifurcation functional $F$ in (3.10) for the nonaxisymmetric case, one can construct the following bifurcation functional

$$
\begin{array}{r}
J[\lambda ; V(\xi), W(\xi)]=\frac{1}{2} \int_{0}^{1}\left[\hat{A}_{11}\left(V_{, \xi \xi}\right)^{2}+2 \hat{A}_{12} V_{, \xi \xi} V_{, \xi}+2 \hat{A}_{13} V_{, \xi \xi} V+\hat{A}_{22}\left(V_{, \xi}\right)^{2}\right. \\
\left.+2 \hat{A}_{23} V_{, \xi} V+\hat{A}_{33}(V)^{2}+\hat{A}_{44}\left(W_{, \xi}\right)^{2}+2 \hat{A}_{45} W_{, \xi} W+\hat{A}_{55}(W)^{2}\right] d \xi,
\end{array}
$$

where the coefficients $\hat{A}_{i j}$ are now given by

$$
\begin{aligned}
& \hat{A}_{11}=\left(C_{\langle 1313\rangle}+\frac{\sigma_{\langle 11\rangle}-\sigma_{\langle 33\rangle}}{2}\right) e^{-4 \varepsilon \xi}, \\
& \hat{A}_{12}=\varepsilon\left[-2\left(C_{\langle 1313\rangle}+\frac{\sigma_{\langle 11\rangle}-\sigma_{\langle 33\rangle}}{2}\right) e^{-4 \varepsilon \xi}\right], \\
& \hat{A}_{13}=\varepsilon^{2}\left(C_{\langle 1313\rangle}+\frac{\sigma_{\langle 11\rangle}-\sigma_{\langle 33\rangle}}{2}\right)\left(\omega r_{i} e^{-6 \xi}\right)^{2},
\end{aligned}
$$




$$
\begin{aligned}
& \hat{A}_{22}=\varepsilon^{2}\left[\left(C_{\langle 1111\rangle}-2 C_{\langle 1133\rangle}+C_{\langle 3333\rangle}+\sigma_{\langle 11\rangle}-\sigma_{\langle 33\rangle}\right)\left(\omega r_{i} e^{-\varepsilon \xi}\right)^{2}\right. \\
& \left.+4\left(C_{\langle 1313\rangle}+\frac{\sigma_{\langle 11\rangle}-\sigma_{\langle 33\rangle}}{2}\right) e^{-4 \varepsilon \xi}\right] \\
& \hat{A}_{23}=\varepsilon^{3}\left(C_{\langle 1122\rangle}+C_{\langle 1133\rangle}-C_{\langle 1111\rangle}-C_{\langle 2233\rangle}-2 C_{\langle 1313\rangle}+\sigma_{\langle 22\rangle}\right.
\end{aligned}
$$$$
\left.+\sigma_{\langle 33\rangle}-2 \sigma_{\langle 11\rangle}\right)\left(\omega r_{i} e^{-\varepsilon \xi}\right)^{2}
$$

$$
\begin{aligned}
\hat{A}_{33}=\varepsilon^{4}\left[\left(C_{\langle 1111\rangle}-2 C_{\langle 1122\rangle}+C_{\langle 222\rangle\rangle}\right.\right. & \left.+2\left(\sigma_{\langle 11\rangle}-\sigma_{\langle 22\rangle}\right)\right)\left(\omega r_{i} e^{-\varepsilon \xi \xi}\right)^{2} \\
& \left.+\left(C_{\langle 1313\rangle}+\frac{\sigma_{\langle 33\rangle}-\sigma_{\langle 11\rangle}}{2}\right)\left(\omega r_{i}\right)^{4}\right]
\end{aligned}
$$

$\hat{A}_{44}=\varepsilon^{2}\left(C_{\langle 1212\rangle}+\frac{\sigma_{\langle 11\rangle}-\sigma_{\langle 22\rangle}}{2}\right)$,

$\hat{A}_{43}=-\varepsilon^{3}\left(C_{\langle 1212\rangle}+\frac{\sigma_{\langle 11\rangle}-\sigma_{\langle 22\rangle}}{2}\right)$,

$\hat{A}_{55}=\varepsilon^{4}\left[C_{\langle 1212\rangle}+\frac{\sigma_{\langle 11\rangle}-\sigma_{\langle 22\rangle}}{2}+\left(C_{\langle 2323\rangle}+\frac{\sigma_{\langle 33\rangle}-\sigma_{\langle 22\rangle}}{2}\right)\left(\omega r_{i} e^{e \xi}\right)^{2}\right]$.

In a similar fashion, for the axisymmetric mode of deformation the modes to be excluded are the rigid body modes as well as the plane strain axisymmetric mode that corresponds to maximum pressure (since a volume controlled experiment is considered here); namely,

$$
\begin{aligned}
& \left(\Delta \dot{u}_{\langle 1\rangle}, \Delta \dot{u}_{\langle 2\rangle}, \Delta \dot{u}_{\langle 3\rangle}\right)_{R . B .}=\left\{\begin{array}{l}
(0, r, 0) \\
(0,0,1),
\end{array}\right. \\
& \left(\Delta \dot{u}_{\langle 1\rangle}, \Delta \dot{u}_{\langle 2\rangle}, \Delta \dot{u}_{\langle 3\rangle}\right)_{M, P}=\left(\frac{1}{r}, 0,0\right) .
\end{aligned}
$$

Hence, the essential boundary conditions for the admissible functions of the functional $J$ in (3.15) are

$$
V_{, \xi}(0)=0, \quad V(0)=0, \quad W(0)=0 .
$$

Having established the appropriate bifurcation functionals for the problem, i.e., the functionals which are quadratic in the eigenmode and whose first variation (i.e., $\delta F=0$ for $n \neq 0, \delta J=0$ for $n=0$ ) is the weak form of the bifurcation equations, attention is turned to the choice of the load parameter $\lambda$.

Two different definitions, according to the type of loading, will be used for the load parameter $\lambda$. For the case of an internally pressurized cylinder, i.e., $p(0)=p_{i}, p(1)=0$, 


$$
\lambda \equiv r_{0} / R_{0} \quad \text { (int. press.) }
$$

while for the externally pressurized case, i.e., $p(0)=0, p(1)=p_{0}$,

$$
\lambda \equiv r_{i} / R_{i} \quad \text { (ext. press.). }
$$

Two additional sets of relations will be also recorded here for future use. Firstly, in addition to the current thickness parameter $\varepsilon \equiv \ln \left(r_{0} / r_{i}\right)$ the initial thickness parameter $E \equiv \ln \left(R_{0} / R_{i}\right)$ is defined. From the incompressibility and plane strain conditions of the prebifurcation configuration one has

$$
R_{0}^{2}-R_{i}^{2}=r_{0}^{2}-r_{i}^{2}
$$

which, in conjunction with (3.19) or (3.20) and the definition of the initial and current thickness parameters, yields the relations

$$
\begin{array}{ll}
\varepsilon=-\frac{1}{2} \ln \left(1+\frac{e^{-2 E}-1}{\lambda^{2}}\right) & \text { (int. press.), } \\
\varepsilon=\frac{1}{2} \ln \left(1+\frac{e^{2 E}-1}{\lambda^{2}}\right) \quad \text { (ext. press.). }
\end{array}
$$

Secondly, and in a similar fashion as for the initial thickness parameter, a nondimensional initial axial wave number $\Omega$ is defined by $\Omega=\omega R_{i}$ which, with the help of (3.22) and (3.19), (3.20), is related to the non-dimensional current axial wave number $\alpha r_{i}$ by

$$
\begin{array}{ll}
\omega r_{i}=\Omega \lambda e^{E-\varepsilon} & \text { (int. press.), } \\
\omega r_{i}=\Omega \lambda & \text { (ext. press.). }
\end{array}
$$

Having completed the general formulation of the bifurcation problem one moves to the determination of the critical load parameter $\lambda_{c r}$ which is found as follows. Let $\lambda_{c r}$ be the minimum (maximum) value of $\hat{\lambda}\left(\omega r_{i}, n ; \varepsilon\right)$ over all possible values of $\omega r_{i}$ and $n$, where $\hat{\lambda}\left(\omega r_{i}, n ; \varepsilon\right)$ is the minimum (maximum) value of the load parameter for which the bifurcation functional $F$ (if $n \neq 0$ ) or $J$ (if $n=0$ ) loses its positive definiteness under inside (outside) pressurization. This value of $\lambda_{c r}$ corresponds to the first instability occurring in the cylinder as the loading increases from the initial stress free configuration. Also note that the increase (decrease) of $\lambda$ away from $\lambda=1$ in the case of internal (external) pressurization corresponds to an internal (external) volume controlled experiment. One can show without difficulty that the quadratic functionals $F$ (or $J$ ) in $v_{r}, v_{z}$ (or $V, W$ ) respectively, which are continuous functions of $\lambda$, are positive definite for $\lambda=1$ (stress-free state) while at the point of loss of their positive definiteness they satisfy the weak form of the bifurcation equations $\delta F=0$ (or $\delta J=0$ ).

The fact that the present formulation covers all possible solutions of the pressurized infinite cylinder bifurcation problem, i.e., that the set of eigenmodes considered here is adequate for reaching all the eigenvalues of the initial problem stated in (3.1)-(3.4), although it can be proved, will not be addressed here as being a technical mathematical issue outside the scope of the present investigation. 
Since interest centers on the dependence of the critical load and corresponding eigenmode on the shell's thickness, the following asymptotic expansions with respect to the current thickness parameter $\varepsilon$ will be adopted

$$
\begin{array}{ll}
v_{r}=\stackrel{0}{v}_{r}(\xi)+\varepsilon v_{r}(\xi)+\varepsilon^{2} v_{r}(\xi)+\cdots & \left(V=\stackrel{0}{V}(\xi)+\varepsilon V(\xi)+\varepsilon^{2} V(\xi)+\cdots\right), \\
v_{z}=\stackrel{0}{v}_{z}(\xi)+\varepsilon v_{r}(\xi)+\varepsilon^{2} v_{r}(\xi)+\cdots & \left(W=\stackrel{0}{W}(\xi)+\varepsilon W(\xi)+\varepsilon^{2} W(\xi)+\cdots\right), \\
\hat{\lambda}\left(\omega r_{i}, n ; \varepsilon\right)=\hat{\lambda}_{0}\left(\omega r_{i}, n\right)+\varepsilon \hat{\lambda}_{1}\left(\omega r_{i}, n\right)+\varepsilon^{2} \hat{\lambda}_{2}\left(\omega r_{i}, n\right)+\cdots, \\
\lambda_{c r}=\lambda_{0}+\varepsilon \lambda_{1}+\varepsilon^{2} \lambda_{2}+\cdots .
\end{array}
$$

Here asymptotic expansions with respect to the current thickness parameter $\varepsilon$, instead of the physically more meaningful initial thickness parameter $E$ will be considered in view of the resulting algebraic simplicity.

The formulation of the cylinder problem will end with the calculation of the zeroth order term $\hat{\lambda}_{0}\left(\omega r_{i}, n\right)=\hat{\lambda}\left(\omega r_{i}, n ; 0\right)$ in the critical load parameter and the zeroth eigenmode for an arbitrary material. Starting with the nonaxisymmetric mode case $n>1$ and expanding the coefficients $\bar{A}_{i j}$ of the bifurcation functional $F$ with respect to $\varepsilon$, namely,

$$
\bar{A}_{i j}=\overline{\bar{A}}_{i j}+\varepsilon \overline{\bar{A}}_{i j}+\varepsilon^{2} \overline{\bar{A}}_{i j}+\cdots \quad 1 \leqslant i, j \leqslant 5,
$$

one obtains, by using (3.24) and (3.25) in the bifurcation equation $\delta F=0$, the following results by collecting the terms of the like order in $\varepsilon$. The $O(1)$ term gives, assuming $\stackrel{0}{A}_{11} \neq 0$,

$$
{\stackrel{0}{v_{r, \xi \xi}}}=0 .
$$

Using the above result in the $O(\varepsilon)$ term of the bifurcation equations gives

$$
\stackrel{0}{\bar{A}_{11}}{\stackrel{1}{v_{r, \xi \xi}}}+\stackrel{1}{\bar{A}}_{14}{\stackrel{0}{v_{z, \xi}}}^{0}=0
$$

Continuing with the $O\left(\varepsilon^{2}\right)$ term of $\delta F=0$ and employing (3.26) and (3.27), one deduces for $\bar{A}_{1,} \bar{A}_{44}-\left(\frac{1}{\bar{A}} 14\right)^{2} \neq 0, \overline{\bar{A}}_{22} \neq 0$ that

$$
\begin{aligned}
& {\stackrel{0}{v_{r, \xi}}}=0, \quad{\stackrel{1}{v_{r, \xi \xi}}}=0, \quad{\stackrel{0}{v_{z, \xi}}}=0,
\end{aligned}
$$

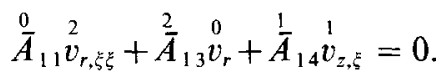

Considering subsequently the $O\left(\varepsilon^{3}\right)$ term and making use also of (3.26)-(3.28), one has the following relations for $v_{r, \xi \xi}^{2}, v_{r, \xi}, \stackrel{0}{v}_{r}, v_{z, \xi}, \stackrel{0}{v_{z}}$

$$
\begin{gathered}
\stackrel{1}{A}_{14} \vec{v}_{r, \xi \xi}+\stackrel{3}{A}_{34} \hat{v}_{r}+\bar{A}_{44}{\stackrel{1}{v_{z, \xi}}}^{2}=0, \\
\stackrel{2}{A}_{22} v_{r, \xi}+\stackrel{3}{A}_{23}{ }^{0} v_{r}+\stackrel{3}{A}_{25}^{0} v_{z}=0,
\end{gathered}
$$

while from the $O\left(\varepsilon^{4}\right)$ term of $\delta F=0$ and after considering (3.26)-(3.28) the following two additional relations for $v_{r, \zeta \zeta}^{2}, v_{r, \zeta}, v_{r}, v_{z, \zeta}, v_{z}$ are deduced 


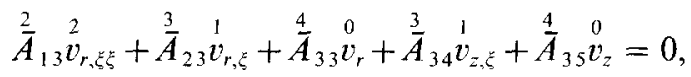

$$
\begin{aligned}
& \bar{A}_{2,5}{ }^{1} v_{r, \xi}+\bar{A}_{35}{ }^{0} v_{r}+\bar{A}_{55}{ }^{0} v_{z}=0 .
\end{aligned}
$$

Hence, a non-trivial solution to the homogeneous linear system (3.28) ${ }_{2},(3.29),(3.30)$ exists if the following condition is satisfied:

$$
\begin{aligned}
& \frac{\left(\bar{A}_{13}\right)^{2} \bar{A}_{44}+\left(\bar{A}_{34}\right)^{2} \bar{A}_{11}-2 \bar{A}_{13} \bar{A}_{14} \bar{A}_{34}}{\bar{A}_{11} \bar{A}_{44}-\left(\bar{A}_{14}\right)^{2}}+\frac{\left(\frac{3}{\bar{A}_{23}}\right)^{2} \bar{A}_{55}+\left(\frac{4}{A}_{35}\right)^{2} \bar{A}_{22}-2 \bar{A}_{23} \bar{A}_{25} \bar{A}_{35}}{\bar{A}_{22} \bar{A}_{55}-\left(\bar{A}_{25}\right)^{2}} \\
& -\bar{A}_{33}=0 .
\end{aligned}
$$

In the derivation of (3.31) it was tacitly assumed that $\bar{A}_{1 \mathrm{I}} \neq 0, \bar{A}_{11} \overline{\bar{A}}_{44}-\left(\frac{1}{\bar{A}_{14}}\right)^{2} \neq 0$, $\bar{A}_{22}{ }^{4} \bar{A}_{55}-\left(\bar{A}_{25}\right)^{2} \neq 0$ while use was also made of the property that all the $\bar{A}_{i j}$ terms entering (3.31), which incidentally are the lowest nonzero terms in the asymptotic expansions of each $\bar{A}_{i j}$ in (3.11), are independent of $\xi$ as one can easily show. Since each of the aforementioned coefficients is a function of $\hat{\lambda}_{0}\left(\omega r_{i}, n\right)$, the solution of (3.31) provides the first term in the expansion of the critical load $\hat{\lambda}\left(\omega r_{i}, n ; \varepsilon\right)$ as a function of the axial and circumferential wave numbers $\omega r_{i}$ and $n(n>1)$. For the special case of $n=1$, the essential boundary condition (3.13) has also to be considered in the analysis. In this case and from the requirement $\hat{v}_{r}=0$ the $0\left(\varepsilon^{2}\right)$ terms yield (for $\left.\stackrel{0}{A}_{11} \neq 0, \bar{A}_{11} \overline{\bar{A}}_{44}-\left(\frac{1}{A}_{14}\right)^{2} \neq 0\right)$

$$
\frac{2}{A}_{22} \stackrel{4}{A}_{55} \quad\left(\bar{A}_{25}\right)^{2}=0
$$

which is the condition providing $\hat{\lambda}_{0}\left(\omega r_{i}, 1\right)$. For the case of $n=0 \dagger$ a similar asymptotic analysis for $J=0$ yields the following equation for $\hat{\lambda}_{0}\left(\omega r_{i}, 0\right)$

$$
\bar{A}_{11}=0 \text {. }
$$

Once $\hat{\lambda}_{0}$ is found, the critical axial $\left(\omega r_{i}\right)$ and circumferential $(n)$ wavenumbers i.e., those which minimize or maximize-according to the type of loading- $\hat{\lambda}_{0}\left(\omega r_{i, n} n\right)$ can be established. For higher order terms in the expansion for $\hat{\lambda}$ and their corresponding eigenmodes the general expressions for the arbitrary material [analogous to (3.31)(3.37)] are extremely involved. Hence these higher order calculations will be presented only for the two specific materials considered in this work, in which case they are considerably simpler than the ones required for the general case. It should be noted here that for the special case of hyperelastic materials and using an entirely different approach based on the nonlinear membrane equations bifurcation equations (for the membrane mode) analogous to (3.31) have already been presented in the literature; for more information the interested reader is referred to HAUGHTON and OGDEN (1979) and the references quoted therein.

$\uparrow$ Note: If a pressure controlled experiment is considered i.e., if the essential boundary condition $V(0)=0$ is relaxed, the equation for $\hat{\lambda}_{0}\left(\omega r_{i}, 0\right)$ is $\bar{A}_{11} \bar{A}_{33}-\left(\bar{A}_{13}\right)^{2}=0$ from which the maximum pressure condition is recovered. 
For the higher order terms a mode orthogonality condition is required. To this end the following relations are adopted $(i \neq 0)$

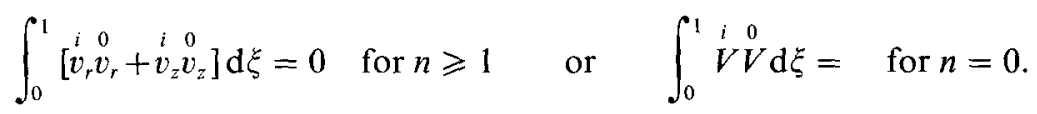

It is understood that the above selection is not unique. This section will end with a remark on the dependence of the critical axial wave number on the cylinder's thickness. From the bifurcation equations $\delta F=0$ or $\delta J=0$, one obtains $\hat{\lambda}\left(\omega r_{i}, n ; \varepsilon\right)$ which, expanded-for a given $\omega r_{i}$ and $n$-about $\varepsilon=0$ yields the expression stated in (3.24) $)_{3}$. But at the critical load $\lambda_{c r}$ the corresponding value of the axial wave number $\left(\omega r_{i}\right)_{c r}(n ; \varepsilon)$ satisfies

$$
\partial \hat{\lambda} /\left.\partial\left(\omega r_{i}\right)\right|_{\left(\omega r_{i}\right)_{r}}=0
$$

which provides, in an implicit form, the functional dependence of $\left(\omega r_{i}\right)_{c r}$ on $\varepsilon$, i.e., $\left(\omega r_{i}\right)_{c r}(n ; \varepsilon)$. From $(3.24)_{3}$ and (3.35) the expansion of the critical axial wave number $\left(\omega r_{i}\right)_{c r}$ about $\varepsilon=0$ gives

$$
\left(\omega r_{i}\right)_{c r}=\omega_{0}+\varepsilon \omega_{1}+\cdots ; \quad \frac{\partial \hat{\lambda}_{0}}{\partial \omega_{0}}=0, \quad \omega_{1}=-\frac{\partial \hat{\lambda}_{1} / \partial \omega_{0}}{\partial^{2} \hat{\lambda}_{0} / \partial \omega_{0} \partial \omega_{0}}
$$

while the critical load $\lambda_{c r}$ takes the form

$$
\begin{aligned}
\lambda_{c r}=\lambda_{0}+\varepsilon \hat{\lambda}_{1}+\varepsilon^{2} \lambda_{2}+\cdots ; \quad \lambda_{0}= & \hat{\lambda}_{0}\left(\omega_{0}, n_{c}\right), \quad \lambda_{1}=\hat{\lambda}_{1}\left(\omega_{0}, n_{c}\right), \\
& \lambda_{2}=\hat{\lambda}_{2}\left(\omega_{0}, n_{c}\right)-\frac{1}{2} \frac{\partial \hat{\lambda}_{1}\left(\omega_{0}, n_{c}\right) / \partial \omega_{0}}{\partial^{2} \hat{\lambda}_{0}\left(\omega_{0}, n_{c}\right) / \partial \omega_{0} \partial \omega_{0}},
\end{aligned}
$$

where all the derivatives of $\hat{\lambda}_{i}$ are evaluated at $\omega r_{i}=\omega_{0}$.

The specialization of the general asymptotic analysis for the cylinder stability problem presented here will be applied subsequently to the two different constitutive laws mentioned at the beginning of this section.

\section{Elastoplastic Cylinder (Power law Type Material)}

As a model of a rate independent elastic-plastic material the hypoelastic material of STören and Rice (1975) will be employed here. The fact that this constitutive choice is a reasonable one for metal plasticity will be established next, together with the derivation of explicit formulas for the prebifurcation stress state for the pressurized cylinder.

\section{Prebifurcation siress state and incremental moduli}

From the kinematics of the prebifurcated solution (i.e. plane strain and incompressibility), the principal stretch ratios of a material point, whose distances from the 
cylinder axis in the initial (stress free) and current configuration are denoted by $R$ and $r$ respectively, are given by

$$
\lambda_{1}=\frac{\mathrm{d} r}{\mathrm{~d} R}=\frac{\left[1+\lambda^{2}\left(e^{2(x-\varepsilon)}-1\right)\right]^{1 / 2}}{\lambda e_{e}^{(x-\varepsilon)}}, \quad \lambda_{2}=\frac{r}{R}=\frac{1}{\lambda_{1}}, \quad \lambda_{3}=1 \quad \text { (int. press.) }
$$

for the case of internal pressurization and by

$$
\lambda_{1}=\frac{\mathrm{d} r}{\mathrm{~d} R}=\frac{\left[1+\lambda^{2}\left(e^{2 x}-1\right)\right]^{1 / 2}}{\lambda e^{x}}, \quad \lambda_{2}=\frac{r}{R}=\frac{1}{\lambda_{1}}, \quad \lambda_{3}=1 \quad \text { (ext. press.) }
$$

for the case of external pressurization. Since in the prebifurcation state, the principal axes of strain remain fixed with respect to the material, the final Cauchy stress depends on the final logarithmic strain as discussed by STÖREN and RICE (1975) and hence the corresponding principal stress-principal strain relation is

$$
\ln \lambda_{i}=\frac{3}{2} \frac{s_{\langle i i\rangle}}{E_{s}} \quad \text { (no sum) } ; \quad s_{\langle i j\rangle}=\sigma_{\langle i j\rangle}-\frac{1}{3} \sigma_{\langle k k\rangle} \delta_{\langle i j\rangle}
$$

where $\sigma_{\langle i i\rangle}$ and $\ln \lambda_{i}$ are the principal values of the Cauchy stress and logarithmic strain tensors respectively, $s_{\langle i j\rangle}$ are the physical components of the Cauchy stress deviator and $E_{s}$ is the secant modulus of the uniaxial stress-strain curve, evaluated at the equivalent stress level $\sigma_{e}$, with

$$
\sigma_{e}^{2}=\frac{3}{2} s_{\langle i\rangle\rangle} S_{\langle i\rangle\rangle}
$$

The equivalent uniaxial stress-strain $\left(\sigma_{e}-\varepsilon_{e}\right)$ relation is here taken to be the power law

$$
\varepsilon_{e}=\frac{\sigma_{y}}{E}\left(\frac{\sigma_{e}}{\sigma_{y}}\right)^{m} \quad m \geqslant 1,
$$

where $E_{\dagger}^{\dagger}$ is the solid's Young's modulus, $m$ is its hardening exponent and $\sigma_{y}$ the uniaxial yield stress. From (4.5) the secant and tangent moduli, $E_{s}$ and $E_{t}$, of the material are found to be

$$
E_{s}=\frac{\sigma_{e}}{\varepsilon_{e}}=E\left(\frac{\sigma_{e}}{\sigma_{y}}\right)^{1-m}, \quad E_{t}=\frac{d \sigma_{e}}{d \varepsilon_{e}}=\frac{E}{m}\left(\frac{\sigma_{e}}{\sigma_{y}}\right)^{1-m} .
$$

From the only non-trivial equilibrium equation for the prebifurcation state (3.9), the following results are deduced for the stress distribution (obviously $\sigma_{\langle i j\rangle}=0$ for $i \neq j)$

$\dagger$ Note: The Young's modulus $E$ should not be confused with the initial thickness parameter $E$ defined in Section 3. 
$\sigma_{\langle 11\rangle}=-\left(\frac{2}{\sqrt{3}} \sigma_{y}\right)\left(\frac{2 E}{\sqrt{3} \sigma_{y}}\right)^{1 / m} \int_{\ln \lambda}^{\ln \lambda+(x-\varepsilon)-0.5 \ln \left[1+\lambda^{2}\left(e^{2(x-s)}-1\right)\right]}\left\{y^{1 / m} /\left[e^{2 y}-1\right]\right\} \mathrm{d} y$,

$\sigma_{\langle 22\rangle}=\sigma_{\langle 11\rangle}+\left(\frac{2}{\sqrt{3}} \sigma_{y}\right)\left(\frac{2 E}{\sqrt{3} \sigma_{y}}\right)^{1 / m}\left\{\ln \lambda+(x-\varepsilon)-0.5 \ln \left[1+\lambda^{2}\left(e^{2(x-\varepsilon)}-1\right)\right]\right\}^{1 / m}$,

$\sigma_{\langle 33\rangle}=\frac{\sigma_{\langle 11\rangle}+\sigma_{\langle 22\rangle}}{2}, \quad p_{t}=-\sigma_{\langle 11\rangle}(0), \quad p_{0}=-\sigma_{\langle 11\rangle}(\varepsilon)=0$

for the internal pressure case, while for the external pressure loading one obtains the following stress state:

$$
\begin{aligned}
& \sigma_{\langle 11\rangle}=-\left(\frac{2}{\sqrt{3}} \sigma_{y}\right)\left(\frac{2 E}{\sqrt{3} \sigma_{y}}\right)^{1 / m} \int_{\ln \lambda}^{\ln \lambda+x-0.5 \ln \left[1+\lambda^{2}\left(e^{2 x}-1\right)\right]}\left\{(-y)^{1 / m} /\left[1-e^{2 y}\right]\right\} \mathrm{d} y, \\
& \sigma_{\langle 22\rangle}=\sigma_{\langle 11\rangle}-\left(\frac{2}{\sqrt{3}} \sigma_{y}\right)\left(\frac{2 E}{\sqrt{3} \sigma_{y}}\right)^{1 / m}\left\{0.5 \ln \left[1+\lambda^{2}\left(e^{2 x}-1\right)\right]-x-\ln \lambda\right\}^{1 / m}, \\
& \sigma_{\langle 11\rangle}=\frac{\sigma_{\langle 11\rangle}+\sigma_{\langle 22\rangle}}{2}, \quad p_{i}=-\sigma_{\langle 11\rangle}(0)=0, \quad p_{0}=-\sigma_{\langle 11\rangle}(\varepsilon) .
\end{aligned}
$$

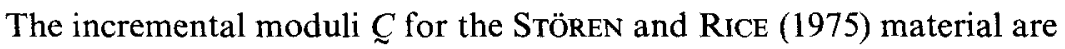

$$
C_{\langle i j k l\rangle}=\frac{2}{3} E_{s}\left[\frac{1}{2}\left(\delta_{i k} \delta_{j l}+\delta_{j k} \delta_{i l}\right)-\frac{3}{2}\left(1-\frac{E_{z}}{E_{s}}\right) \frac{s_{\langle i j\rangle} s_{\langle k l\rangle}}{\sigma_{e}^{2}}\right] .
$$

A minimum requirement for the above solution to be a possible one for an elastoplastic cylinder is that the total loading criterion should be satisfied everywhere in the prebifurcation state of the solid, i.e.,

$$
s_{\langle i j\rangle} D_{\langle i j\rangle} \geqslant 0 ; \quad D_{\langle i j\rangle}=\left(\dot{\lambda}_{k} / \lambda_{k}\right) \delta_{i j} \quad \text { (no sum), }
$$

where $\underset{\sim}{D}$ is the strain rate tensor. From (4.1), (4.2) and (4.7), (4.8), one can easily verify that $(4.10)$ is always satisfied. Consequently, since the plastic (or total) loading criterion (4.10) holds everywhere in the prebifurcation state of the solid, the critical loads to be found from the proposed stability analysis are, at worst, lower bounds for the elastoplastic bifurcation problem of a solid whose total loading incremental moduli are given by (4.9). (Sce Hill (1958) and HuTCHINSON (1974) for the pertaining detailed theory.)

For the inside pressurized cylinder made of the power law material, two values of the load parameter $\lambda$ are of special interest: the one corresponding to maximum pressure and the other corresponding to the loss of ellipticity of the material. The value of the load parameter $\lambda$ corresponding to the maximum inside pressure for a cylinder of initial thickness parameter $E$, say $\lambda_{p}$, is deduced from the requirement $\mathrm{d} \sigma_{\langle 11\rangle}(0) / \mathrm{d} \lambda=0$. Using $(4.7)_{1}$ in conjunction with $(3.22)_{1}$, one finds that $\lambda_{p}$ is given by 


$$
\frac{\ln \left[1+\left(\lambda_{p}^{2}-1\right) e^{2 E}\right]}{\ln \left[\lambda_{p}^{2}\right]}=\left[\frac{1+\left(\lambda_{p}^{2}-1\right) e^{2 E}}{\lambda_{p}^{2}}\right]^{m} .
$$

Since $\lambda_{p}$ is a function of the initial thickness parameter $E$, a straightforward asymptotic expansion about $E=0$ yields

$$
\lambda_{p}=e^{1 / 2 m}-E \sinh \left(\frac{1}{2 m}\right)+\cdots .
$$

In the present work, of interest in the bifurcation analysis of the solid are the bifurcation modes occurring while all the points in the prebifurcation state are in the elliptic regime of the material, i.e. before any type of localized deformation failure occurs in the structure. For the plane strain deformations of the STÖREN and RICE (1975) material, ellipticity is lost when the maximum principal stretch ratio of a material point reaches a strain level of $\max \left(\lambda_{1}, \lambda_{2}\right)=\exp (\sqrt{m-1} / m)$ where $m$ is the solid's hardening exponent. Since in the case of the internally pressurized tube, the maximum principal stretch ratio is the one in the hoop direction at the inner surface of the tube, i.e. $\lambda_{2}(0)$, from (4.1) and using also $(3.22)_{1}$ one finds that for the prebifurcation solution to be entirely in the elliptic range the load parameter $\lambda$ cannot exceed a certain maximum value $\lambda_{E}$ :

$$
\lambda \leqslant \lambda_{E} \equiv\left\{1+\left[\exp \left(2 \frac{\sqrt{m-1}}{m}\right)-1\right] \exp (-2 E)\right\}^{1 / 2} .
$$

As one can see from (4.12) and (4.13), the maximum pressure and the loss of ellipticity in the prebifurcation configuration do occur at substantial strain levels (for mild steel for example $m \simeq 4$ ) and hence these phenomena will be of concern only in the internally pressurized cylinder case, where the critical strains at bifurcation are of comparable order of magnitude.

\section{Elastoplastic cylinder-internal pressure}

Since the critical strains for the internally pressurized cylinder are expected to be well into the material's plastic region, only values of the hardening exponent $m>1$ will be considered. More specifically, and for reasons to be subsequently explained, it will be assumed that $m>2$. Given that for most structural metals this inequality is satisfied (e.g. brass $m \approx 2.5$, steel $m \approx 4$ ), the aforementioned restriction is not very important.

At first, attention is focussed on the determination of the lowest order term in the critical load expansion, i.e., $\hat{\lambda}_{0}\left(\omega r_{i}, n\right)$. For $n \geqslant 1$ and using (4.7), (4.9) into (3.11), one has

$$
\begin{aligned}
& \stackrel{0}{A}_{11}=G_{s}(1-2 y) \frac{1}{n^{2}} \\
& \overline{\bar{A}}_{13}=G_{s}(1-2 y) \frac{n^{2}-1}{n^{2}}
\end{aligned}
$$




$$
\begin{aligned}
& \frac{1}{A}_{14}=G_{s}(1-2 y) \frac{\left(-\omega r_{i}\right)}{n^{2}}, \\
& \bar{A}_{22}=G_{s}\left[4\left(\frac{1}{m}-y\right)+(1-y) \frac{\left(\omega r_{i}\right)^{2}}{n^{2}}\right] \\
& \bar{A}_{23}=G_{s}(1-y) \frac{\left(\omega r_{i}\right)^{2}}{n^{2}} \\
& \bar{A}_{25}=G_{s}\left[1-\frac{2}{m}+y-(1-y) \frac{\left(\omega r_{i}\right)^{2}}{n^{2}}\right]\left(\omega r_{i}\right), \\
& \frac{4}{A}_{33}=G_{s}\left[\frac{\left(n^{2}-1\right)^{2}}{n^{2}}+2 y \frac{n^{4}-1}{n^{2}}+(1+y)\left(\omega r_{i}\right)^{2}+(1-y) \frac{\left(\omega r_{i}\right)^{2}}{n^{2}}\right], \\
& \bar{A}_{34}=G_{s}\left[y+(1-2 y) \frac{1}{n^{2}}\right]\left(\omega r_{i}\right) \\
& \bar{A}_{55}=G_{s}\left[(1+y) n^{2}+\left(1+\frac{1}{m}\right)\left(\omega r_{i}\right)^{2}+(1-y) \frac{\left(\omega r_{i}\right)^{4}}{n^{2}}\right], \\
& \bar{A}_{44}=G_{s}\left[1-y+(1-2 y) \frac{\left(\omega r_{i}\right)^{2}}{n^{2}}\right] \\
& \bar{s}_{s}\left[1+y-(1-y) \frac{\left(\omega r_{i}\right)^{2}}{n^{2}}\right]\left(\omega r_{i}\right),
\end{aligned}
$$

where $y \equiv \ln \hat{\lambda}_{0}$ and the common factor $G_{s} \equiv\left(\sigma_{y} / 2 \sqrt{3}\right)\left(2 E / \sqrt{3} \sigma_{y}\right)^{1 / m}\left(\ln \hat{\lambda}_{0}\right)^{(1 / m)-1}$ with $E$ and $\sigma_{y}$ the material's Young's modulus and uniaxial yield stress respectively. Substituting (4.14) into (3.31), one obtains for $n \geqslant 2$ the following equation for $\hat{\lambda}_{0}$

$$
\begin{aligned}
\frac{6}{m} \beta^{2}+ & y\left\{\frac{8}{m}\left(n^{2}-1\right)+\frac{20}{m}\left(n^{2}-1\right) \beta+\left[n^{2}\left(6+\frac{10}{m}\right)-\left(12+\frac{6}{m}\right)\right] \beta^{2}\right. \\
+ & \left.n^{2}\left(3+\frac{1}{m}\right) \beta^{3}\right\}+y^{2}\left\{8\left(\frac{1}{m}-1\right)\left(n^{2}-1\right)+\left(\frac{4}{m}-16\right)\left(n^{2}-1\right) \beta\right. \\
+ & {\left.\left[-n^{2}\left(16+\frac{2}{m}\right)+12\right] \beta^{2}-n^{2}\left(5+\frac{1}{m}\right) \beta^{3}\right\} } \\
+ & y^{3}\left\{-8\left(n^{2}-1\right)-8\left(n^{2}-1\right) \beta+2 n^{2} \beta^{2}+2 n^{2} \beta^{3}\right\}=0,
\end{aligned}
$$

where for simplicity the notation $\beta \equiv\left(\omega r_{i}\right)^{2} / n^{2}$ was introduced. For the particular case $n=1$, from $(4.14)$ in conjunction with $(3.32)$ the following equation for $\hat{\lambda}_{0}$ results 


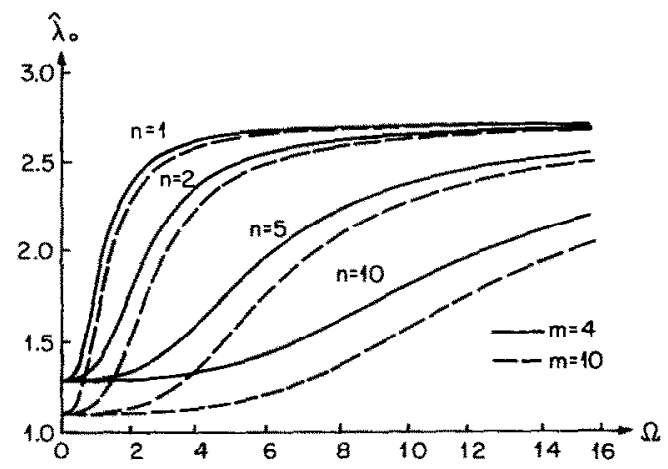

FIG. 1A. Dependence of the zeroth order critical load $\hat{\lambda}_{0}$ on the nondimensional axial wavenumber $\Omega$ for various circumferential wavenumbers $n$ for a power law material with hardening exponents $m=4$ and $m=10$ in the case of inside pressure.

$$
\begin{array}{r}
{\left[\frac{4}{m}+\frac{8}{m} \beta+\left(3+\frac{1}{m}\right) \beta^{2}\right]+y\left[4\left(\frac{1}{m}-1\right)-6 \beta-\left(5+\frac{1}{m}\right) \beta^{2}\right]} \\
+y^{2}\left[-4-2 \beta+2 \beta^{2}\right]=0 .
\end{array}
$$

For the axisymmetric bifurcation modes $n=0$, from (3.33) with the help of (3.16) and (4.7), (4.9), the solution $\ln \hat{\hat{\lambda}}_{0}=1$ is obtained which is unacceptable as violating (4.13) (note that for $E=0, \ln \hat{\lambda}_{E}=\sqrt{m-1} / m<1$ ).

The dependence of the critical load parameter $\hat{\lambda}_{0}$ closest to unity, i.e., the minimum of the roots of $(4.15),(4.16)$ greater than unity, on the circumferential $(n)$ and initial axial $(\Omega)$ wavenumbers recalling from $(3.23)_{2}$ that $\omega r_{i}=\Omega \hat{\lambda}_{0}$ is depicted in Fig. $1 \mathrm{~A}$ in solid lines for the case $m=4$ and dashed lines for $m=10$. The absolute minimum of $\hat{\lambda}_{\theta}$ occurs at the plane strain mode $\Omega=\omega r_{i}=0$ for all circumferential wavenumbers $n$ at $\hat{\lambda}_{0}(0, n)=\exp (1 / m)$ which is past the value $\lambda_{P}(0)$ corresponding to the maximum hydrostatic pressure for the membrane $\lambda_{P}(0)=\exp (1 / 2 m)($ see $(4.12))$ but below the loss of ellipticity limit of $\lambda_{E}(0)=\exp (\sqrt{m-1} / m)$ (see (4.13)) for $m>2$ which justifies restriction on the hardening exponent mentioned earlier.

Since the absolute minimum of $\hat{\lambda}_{0}(\Omega, n)$ is attained at $\Omega \leqslant 0$, and in view of the symmetry of $\hat{\lambda}(\Omega, n ; E)$ with respect to the $\Omega$ axis, from the continuity of $\hat{\lambda}$ in the neighborhood of $E=0$, the absolute minimum of $\hat{\lambda}(\Omega, n ; E)$ will also occur at $\Omega=0$ and hence in the analysis of the higher order terms in the expansion of $\hat{\lambda}$ the plane strain condition $\Omega=\omega r_{i}=0, v_{z}=0$ will be assumed. As explained in Section 3 , this and all subsequent asymptotic analyses will be conducted for simplicity with respect to the current thickness parameter $\varepsilon$ but the results will also be presented in terms of the initial thickness parameter $E$.

Substituting (4.7) and (4.9) into (3.11) and expanding the bifurcation equation $\delta F=0$ (with $F$ given by (3.10)) about $\varepsilon=0$ following (3.24), one obtains, by collecting the terms of the like order in $\varepsilon$ up to the $O\left(\varepsilon^{6}\right)$ term, the following results for $\hat{\lambda}(0, n ; \varepsilon)$, valid for $n \geqslant 1$ : 


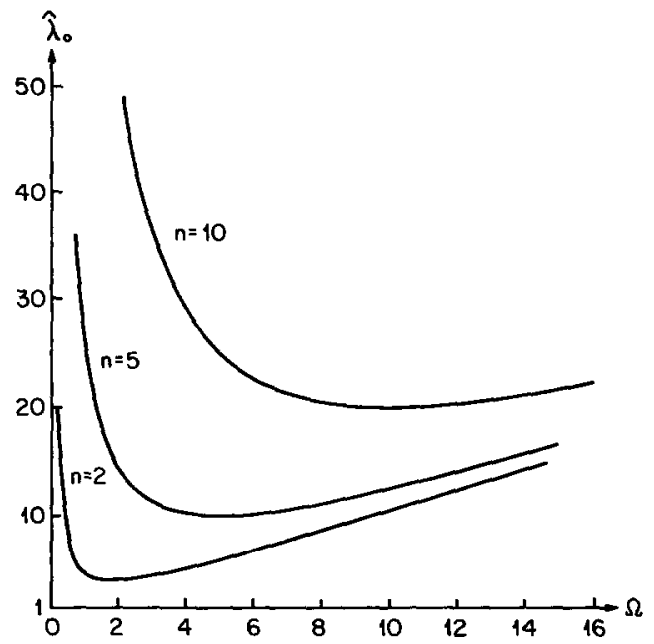

Fig. 1B. Dependence of the zeroth order critical load $\hat{\lambda}_{0}$ on the nondimensional axial wavenumber $\Omega$ for various circumferential wavenumbers $n$ for a neo-Hookean solid in the case of inside pressure.

$$
\begin{aligned}
\hat{\lambda}(0, n ; \varepsilon)=e^{1 / m}-\varepsilon & \frac{e^{1 / m}}{2}\left(e^{2 / m}-1\right) \\
& +\varepsilon^{2} e^{1 / m}\left[\frac{1}{m} \frac{n^{2}-1}{12}+\frac{\left(e^{2 / m}-1\right)^{2}}{12}\left(\frac{5}{2}+m\right)+\frac{e^{2 / m}-1}{6}\right]+\cdots
\end{aligned}
$$

while the corresponding eigenmode $v_{r}(0, n ; \varepsilon ; \xi)$ is

$$
v_{r}(0, n ; \varepsilon ; \xi)=\left\{\begin{array}{l}
0+\varepsilon \xi+\varepsilon^{2} 0+\ldots(n=1) \\
0+\varepsilon\left(\xi-\frac{1}{2}\right)+\varepsilon^{2} \frac{(m+1)}{12}\left(e^{2 / m}-1\right)+\cdots \quad(n>1) .
\end{array}\right.
$$

Note from (4.17), that the critical circumferential wavenumber is $n_{c}=1$ in agreement with the finite element calculations of CHU (1979) and the experiments of LARSON (1979) $\dagger$ who also finds that the critical mode, in internally pressurized copper and aluminum tubes, is one of plane strain with $n_{c}=1$. It should also be remarked here that in CHU's (1979) work, no proof was given to the fact that the critical mode is a plane strain one (the corresponding analysis is from the outset a plane strain one) while the proposed "shell limit" analysis, which uses the rigid-plastic cylinder's buckling mode of StORAKERS (1971), does not predict $n_{c}$ or any higher order term in the expansion of the critical load. Using (3.22) ${ }_{1}$ into (4.17), one finds, recalling that $n_{c}=1$, the following dependence of the critical load $\lambda_{c r}$ on the initial thickness parameter $E$

$$
\lambda_{c r}=e^{1 / m}-E \sinh \left(\frac{1}{m}\right)+E^{2} \frac{e^{-(2 / m)}}{3} \sinh \left(\frac{1}{m}\right)\left[1+\left(\frac{5}{2}+m\right) e^{1 / m} \sinh \left(\frac{1}{m}\right)\right]+\cdots
$$




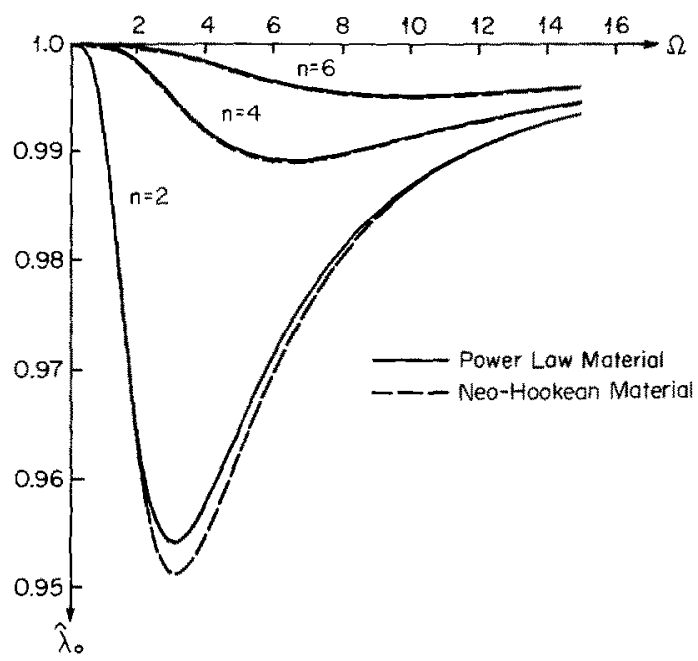

Fic. 2. Dependence of the zeroth order critical load $\hat{\lambda}_{0}$ on the nondimensional axial wavenumber $\Omega$ for various circumferential wavenumbers $n$ for both a power law $(m=1)$ and a neo-Hookean solid in the case of outside pressure.

An analogy can be drawn here between the inside pressurized cylinder and the plane strain tension test in HILL and Hutchinson (1975). In both cases as the thickness tends to zero, the fundamental eigenmode is a membrane one (i.e. the corresponding strains as $\varepsilon \rightarrow 0^{\circ}$ are nonzero) at which all the wavenumbers $n$ are possible while the modes do separate as the thickness increases.

\section{Elastoplastic cylinder-external pressure}

Since buckling in externally pressurized cylindrical shells occurs at small strains, with the critical strains tending to zero as the shell thickness vanishes, a hardening exponent $m=1$ will be assumed for the material in this case.

The determination of $\hat{\lambda}_{0}\left(\omega r_{i}, n\right)$ proceeds exactly as in the previous subsection with the characteristic equations for $\hat{\lambda}_{0}$ being given by (4.15) for $n \geqslant 2$ and by (4.16) for $n=1$. Of interest is again the root closest to unity of the above equations, $\hat{\lambda}_{0}\left(\omega r_{i}, n\right)$, which is given in this case by the maximum (less than unity) root of $(4.15)(n>1)$. The dependence of $\hat{\lambda}_{0}$ on the circumferential $(n)$ and initial axial wavenumber $(\Omega)$, obtained by using $(3.23)_{2}$ into $(4.15)$, is depicted in Fig. 2 . As expected from classical analyses of the problem (see, for example, Tmoshenko and GERE (1961), $\max _{\Omega} \hat{\lambda}_{0}=\hat{\lambda}_{0}(0, n)=1$ for all circumferential wavenumbers $n$ and hence the plane strain mode $\omega r_{i}=\Omega=0$ is the critical one in agreement with the classical analysis of the problem using a nonlinear shell theory (see, for example, TIMOSHENko and GeRE (1961)). Using the same continuity arguments for $\hat{\lambda}(\Omega, n ; E)$ as $E \rightarrow 0$ as in Section 4.2 , the analysis for the higher order terms in the expansion of $\hat{\lambda}$ is performed under the assumption of plane strain conditions $\left(\Omega=\omega r_{i}=0, v_{z}=0\right)$.

Substituting (4.8) into (3.11) and expanding for $n \neq 1$, the bifurcation equation $\delta F=0$ (with $F$ given by (3.10)) about $\varepsilon=0$ following (3.24), one obtains by collecting terms of like order in $\varepsilon$ up to the $O\left(\varepsilon^{8}\right)$ term, the following results for $\hat{\lambda}(0, n ; \varepsilon)$ 


$$
\hat{\lambda}(0, n ; \varepsilon)=1-\varepsilon^{2} \frac{\left(n^{2}-1\right)}{12}-\varepsilon^{3} \frac{\left(n^{2}-1\right)}{12}+\varepsilon^{4} \frac{\left(n^{2}-1\right)\left(39 n^{2}-31\right)}{1440}+\cdots,
$$

while the corresponding terms in the eigenmode expansion of $v_{r}(0, n ; \varepsilon ; \xi)$ are found, with the help of the orthogonality condition (3.34), to be

$$
\begin{aligned}
v_{r}(0, n ; \varepsilon ; \xi)=1+\varepsilon^{2} & \frac{\left(n^{2}-1\right)}{2}\left[-\xi^{2}+\xi-\frac{1}{6}\right]+\varepsilon^{4} \frac{\left(n^{2}-1\right)}{12}\left[-\left(3 n^{2}+1\right)\right. \\
& \left.\times\left(\frac{\xi^{4}}{2}-\xi^{3}+\frac{\xi}{2}-\frac{1}{10}\right)+\frac{n^{2}-1}{2}\left(\xi^{2}-\xi+\frac{1}{6}\right)\right]+\cdots,
\end{aligned}
$$

where in both (4.20) and (4.21) it is assumed that $n>1$.

From (4.20) it follows that the critical circumferential wavenumber $n_{c}=2$. Assuming the Young's modulus to be unity for simplicity, the corresponding critical pressure is found from (4.20) and $(4.8)_{5}$ to be $\left(p_{0}\right)_{c r}=\varepsilon^{3}\left(n^{2}-1\right) / 9 \simeq(h / R)^{3}\left(n^{2}-1\right) / 9$ (where $h \equiv R_{0}-R_{i}, R \equiv\left(R_{0}+R_{i}\right) / 2$ ) which coincides with the classical stability result for the externally pressurized incompressible, isotropic, linearly elastic circular cylinder as one can see, for example, in TIMOSHENKo and GERE (1961).

Expressing the critical load $\lambda_{c r}$ in terms of the initial thickness parameter $E$, one finds from $(3.22)_{2}$ and (4.20) that

$$
\lambda_{c r}=1-E^{2} \frac{\left(n_{c}^{2}-1\right)}{12}-E^{3} \frac{\left(n_{c}^{2}-1\right)}{12}-E^{4} \frac{\left(n_{c}^{2}-1\right)\left(n_{c}^{2}-9\right)}{1440}=1-\frac{E^{2}}{4}-\frac{E^{3}}{4}+\frac{E^{4}}{96}+\cdots .
$$

Again the analogy is drawn here between the outside pressurized cylinder and the plane strain compression test in IIILL and Hutchinson (1975). In both cases as the thickness tends to zero, the fundamental eigenmode is a bending one (i.e. the corresponding critical strains, as $\varepsilon \rightarrow 0$, vanish).

\section{Hyperplastic Cylinder (neo-Hookean Type Material)}

A neo-Hookean constitutive law will be considered here as a model for a rubber-like elastic solid. The reason for this particular choice, besides the resulting considerable algebraic simplifications, lies in the fact that this material never loses ellipticity and that in the internal pressurization case the fundamental solution not only does not present a pressure maximum, but it also exhibits a lowest critical load which does not correspond to a plane strain solution.

\section{Prebifurcation stress state and incremental moduli}

The kinematics of the prebifurcated solution are the same as in the previous section and hence the principal stretch ratios $\lambda_{i}$ are given by (4.1), (4.2). Recalling that for a neo-Hookean material the strain energy density $W$ is given by 


$$
W=\frac{1}{2} G\left(I_{c}-3\right)=\frac{1}{2} \mathrm{G}\left(\lambda_{1}^{2}+\lambda_{2}^{2}+\lambda_{3}^{2}-3\right) \text {, }
$$

where $I_{c}$ is the first invariant of the Cauchy-Green deformation tensor, and that the principal Cauchy stresses for an incompressible hyperelastic material are related to $W$ and the hydrostatic pressure $\bar{p}$ by

$$
\sigma_{\langle i i\rangle}=\lambda_{i} \frac{\partial W}{\partial \lambda_{i}}-\bar{p} \quad \text { (no sum) }
$$

one deduces for the prebifurcation stress state $\sigma_{\langle i j\rangle}$ that

$$
\begin{aligned}
& \sigma_{\langle 11\rangle}=\frac{1}{2} \mathrm{G}\left[\left(\lambda_{1}\right)^{2}-\frac{1}{\lambda^{2}}+\ln \left(\lambda_{1} \lambda\right)^{2}\right], \\
& \sigma_{\langle 22\rangle}=\sigma_{\langle 11\rangle}+\mathrm{G}\left[\left(\lambda_{2}\right)^{2}-\left(\lambda_{1}\right)^{2}\right], \\
& \sigma_{\langle 33\rangle}=\sigma_{\langle 11\rangle}+\mathrm{G}\left[\left(\lambda_{3}\right)^{2}-\left(\lambda_{1}\right)^{2}\right],
\end{aligned}
$$

with $\lambda_{i}$ 's given by (4.1) for the internal and by (4.2) for the external pressure case.

Following HiLl (1969), the physical components of the incremental moduli tensor $C$ are in this case [with $W$ defined in (5.1)] for $i \neq j$ given by

$$
C_{\langle i i i i\rangle}=2 \mathrm{G}\left(\lambda_{i}\right)^{2}, \quad C_{\langle i i j\rangle}=0, \quad C_{\langle i j i j\rangle}=\frac{\mathrm{G}}{2}\left[\left(\lambda_{i}\right)^{2}+\left(\lambda_{j}\right)^{2}\right] \quad \text { (no sum). }
$$

\section{Hyperelastic cylinder-internal pressure}

As in the case of the elastoplastic cylinder, the analysis will start with the determination of the lowest order term in the expansion of the critical load. For $n \geqslant 1$ and using (5.3), (5.4) into (3.11), one obtains

$$
\begin{aligned}
& \bar{A}_{11}=\mathrm{G} \frac{1}{y n^{2}}, \quad \bar{A}_{13}=\mathrm{G} \frac{\left(n^{2}-1\right)}{y n^{2}} \\
& \frac{1}{A}_{14}=\mathrm{G} \frac{\left(-\omega r_{i}\right)}{y n^{2}}, \quad \bar{A}_{22}=\mathrm{G}\left[\frac{3}{y}+y+\frac{\left(\omega r_{i}\right)^{2}}{n^{2}}\right] \\
& \bar{A}_{23}=\mathrm{G} \frac{\left(\omega r_{i}\right)^{2}}{n^{2}}, \quad \bar{A}_{25}=\mathrm{G}\left[y+\frac{\left(\omega r_{i}\right)^{2}}{n^{2}}\right]\left(-\omega r_{i}\right), \\
& \bar{A}_{33}=\mathrm{G}\left[y\left(n^{2}-1\right)-\frac{1}{y} \frac{\left(n^{2}-1\right)}{n^{2}}+\left(\omega r_{i}\right)^{2}+\frac{\left(\omega r_{i}\right)^{2}}{n^{2}}\right], \quad \frac{3}{A_{34}}=\mathrm{G} \frac{\left(\omega r_{i}\right)}{y n^{2}}, \\
& \bar{A}_{35}=\mathrm{G}\left[y-\frac{\left(\omega r_{i}\right)^{2}}{n^{2}}\right]\left(\omega r_{i}\right), \quad \frac{2}{A}{ }_{44}=\mathrm{G} \frac{1}{y}\left[1+\frac{\left(\omega r_{i}\right)^{2}}{n^{2}}\right], \\
& \bar{A}_{55}=\mathrm{G}\left[y n^{2}+(y+1)\left(\omega r_{i}\right)^{2}+\frac{\left(\omega r_{i}\right)^{4}}{n^{2}}\right],
\end{aligned}
$$

+ Note: The constant $G$ is the material's initial shear modulus related to its initial Young's modulus $E$ by $\mathrm{G}=E / 3$. 
where in this case $y \equiv\left(\hat{\lambda}_{0}\right)^{2}$. Upon substitution of (5.5) into the governing equation for $\hat{\lambda}_{0}$, for the case $n \geqslant 2$, the following equation is obtained (recall also the previously used notation $\left.\beta \equiv\left(\omega r_{i}\right)^{2} / n^{2}\right)$ :

$$
\begin{aligned}
3 \beta(1+\beta)\left[n^{2}-1+n^{2} \beta\right]+y\left[3\left(n^{2}-1\right)+3\left(2 n^{2}-1\right) \beta+\left(n^{2}-4\right) \beta^{2}-2 n^{2} \beta^{3}\right] \\
+y^{2}\left[-\left(4 n^{2}+2\right) \beta-\left(4 n^{2}+6\right) \beta^{2}-n^{2} \beta^{3}\right]+y^{3}\left[-2\left(n^{2}-1\right)+\left(6-2 n^{2}\right) \beta-3 n^{2} \beta^{2}\right] \\
+y^{4}\left[-\beta\left(3 n^{2}-1\right)+\beta^{2}\right]+y^{5}\left[-\left(n^{2}-1\right)+\beta\right]=0 .
\end{aligned}
$$

In the special cases $n=1$ and $n=0$ from (5.5) and (3.32), (3.33), one can easily see that no solution for $\hat{\lambda}_{0}$ exists.

The dependence of $\hat{\lambda}_{0}$, i.e., the smallest of the roots of (5.6) which are greater than unity, on the circumferential $(n)$ and initial axial $(\Omega)$ wavenumbers (recalling once more that from $(3.23)_{1} \omega r_{i}=\Omega \hat{\lambda}$ ), is depicted in Fig. 1B. Note that in this case, no instability is possible for the plane strain case $(\Omega=0)$, regardless of the circumferential wavenumber $n$. The critical circumferential wavenumber $n_{c}=2$ while $\lambda_{0}=\min _{\Omega} \hat{\lambda}_{0}\left(\Omega, n_{c}\right)=\hat{\lambda}_{0}\left(\Omega_{0}, 2\right)=3.981$ with the minimum occurring at $\Omega=\Omega_{0}=$ 1.792 (corresponding value of $\omega r_{i}$ is $\omega_{0}=7.135$ ). Using (4.1), (5.3), (5.4), (3.24) into the bifurcation equation $\delta F=0$ (where $F$ is given by (3.10), (3.11)) and expanding about $\varepsilon=0$ up to the $O\left(\varepsilon^{5}\right)$ term, as explained in Section 3, one obtains by employing also (3.35) [recalling (3.36) and (3.37)] after some laborious but straightforward algebraic manipulations

$$
\lambda_{c r}=3.981-\varepsilon 29.561+\cdots ; \quad\left(\omega r_{i}\right)_{c r}=7.135-\varepsilon 3.513+\cdots .
$$

The corresponding eigenmode expansions are found to be

$$
\begin{aligned}
& v_{r}\left(\xi ;\left(\omega r_{i}\right)_{c r}, n_{c} ; \varepsilon\right)=1+\varepsilon(-6.879 \xi+3.435)+\cdots, \\
& v_{z}\left(\xi,\left(\omega r_{i}\right)_{c r}, n_{c} ; \varepsilon\right)=-0.908+c(-7.135 \xi+3.563)+\cdots .
\end{aligned}
$$

Using (3.22), into (5.7) the critical load can also be expressed in terms of the initial thickness parameter $E$ as

$$
\lambda_{c r}=3.981-E 1.865+\cdots ; \quad \Omega_{c r}=1.792-E 0.839+\cdots .
$$

Due to their extreme algebraic complexity the general formulas for $\lambda_{i}, \omega_{i}, i_{r}, i_{z}$ in terms of $\omega r_{i}, n$ will not be recorded here.

\section{Hyperelastic cylinder-external pressure}

Starting with the determination of $\hat{\lambda}_{0}\left(\omega r_{i} n\right)$, which is given by the maximum root of (5.6) less than unity, one obtains results very similar to the power law case. As can be seen from Fig. 2 , where $\hat{\lambda}_{0}$ is plotted versus the initial axial wavenumber $\Omega$, only for $n=2$ and at the vicinity of $\Omega=3$, the power law and neo-Hookean material present their maximum difference and even then this difference is less than $1 \%$.

Again the plane strain mode is the critical one and by proceeding as in Section 4, the expansion of the bifurcation equation $\delta F=0$ up to the $O\left(\varepsilon^{8}\right)$ term produces exactly the same results for the critical loads and eigenmodes as for the power law material. [See equations (4.20)-(4.22).] It was found that only after the $O\left(c^{6}\right)$ terms in 
the aforementioned expansions the effects of the constitutive difference are felt in the corresponding asymptotic results.

\section{Numerical Method and Results}

For the cylindrical shell of arbitrary thickness, the corresponding bifurcation equations (i.e. the Euler equations for the functionals $F$ and $I$ in (3.10) and (3.15) respectively) are extremely cumbersome and no analytical solution seems possible for the general case. Hence a numerical technique is required for the calculation of the critical loads and the corresponding eigenmodes.

\section{F.E.M. Formulation for arbitrarily thick cylinder}

In contrast with the power series expansion methods usually adopted in similar calculations (see HAUGHTON and OGDEN, 1979) and also references quoted therein), the Finite Element Method seems to be the natural candidate for the task in view of the variational formulation of the problem presented in Section 3. The interval $(0,1)$ of the normalized thickness coordinate is subdivided into $N_{e}$ equal subintervals (elements) where depending on the initial thickness parameter $E, N_{e}$ varies between 2 and 20. For $n \neq 0(n=0)$ Hermitian cubics are chosen as shape functions for $v_{r}(V)$ while a linear interpolation is adopted for $v_{z}(W)$ resulting in a three degree-of-freedom per node line structure. A six point Gaussian quadrature is used in the numerical integration involved in the construction of the discretized stiffness matrix $[K(\lambda)]$, whose positive definiteness in terms of $\lambda$ is detected by checking when the minimum entry of the diagonal matrix $(D)$ in the Cholesky decomposition of $(K)$ $\left[(K)=(L)(D)(L)^{T} ;(L)\right.$ a lower diagonal matrix $]$ is nonpositive. A straightforward bisection method is employed for the determination of the critical load $\lambda_{c r}$.

\section{Numerical results}

Starting with the internally pressurized cylinder case, the dependence of the critical load $\lambda_{c r}$ on the nondimensionalized axial wavenumber $\Omega$ has been investigated for different values of the initial thickness parameter $E$. The results for the power law material with hardening exponent $m=4$ and circumferential wavenumber $n=n_{c}=1$ are depicted in Fig. 3A, while the corresponding calculations for the neo-Hookean material with $n=n_{c}=2$ are shown in Fig. 3B. Note that for the power law material the minimum for the critical load $\lambda_{c r}$ always occurs at the plane strain case $\Omega=0$ as discussed in Section 4. Also note in the same figure that only in the interval $|\Omega|<1$ (approximately) a bifurcation solution exists in the elliptic region of the prebifurcation state $\dagger$ (values of $\lambda_{c r}$ below the straight lines $\lambda=\lambda_{E}$ in Fig. $3 \mathrm{~A}$ with $\lambda_{E}$ given by (4.13)). The peculiarity of decreasing critical load (for a given $\Omega$ ) with increasing thickness of

\footnotetext{
+ Note: Numerical calculations using the three-dimensional definition for the loss of ellipticity (as opposed to the plane strain considerations in Section 4) confirm that $\lambda_{E}$ is the lowest load corresponding to the onset of loss of ellipticity in the prebifurcation solution of a cylinder with thickness parameter $E$.
} 


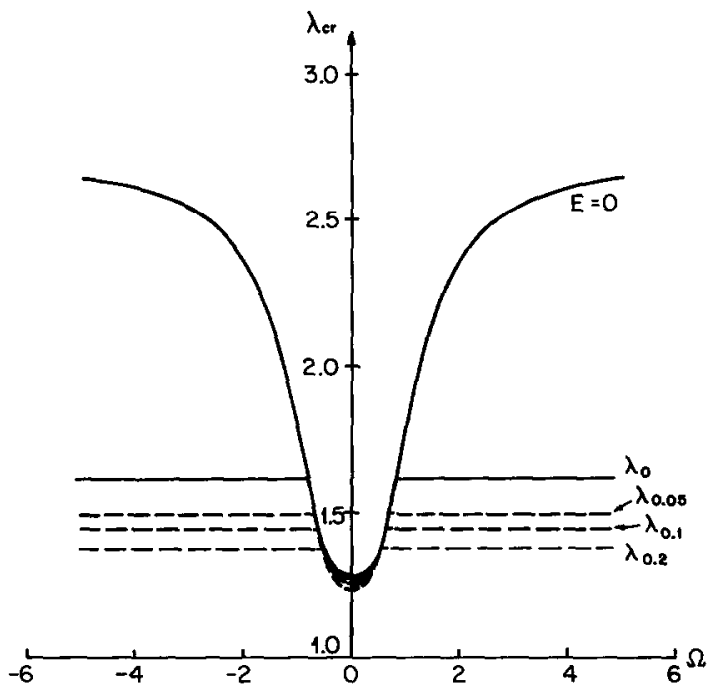

FIG. 3A. Dependence of lowest critical $\lambda_{c r}$ on the nondimensional axial wavenumber $\Omega$ for various values of the initial thickness parameter $E$ in the case of an internally pressurized power law tube with $m=4$.

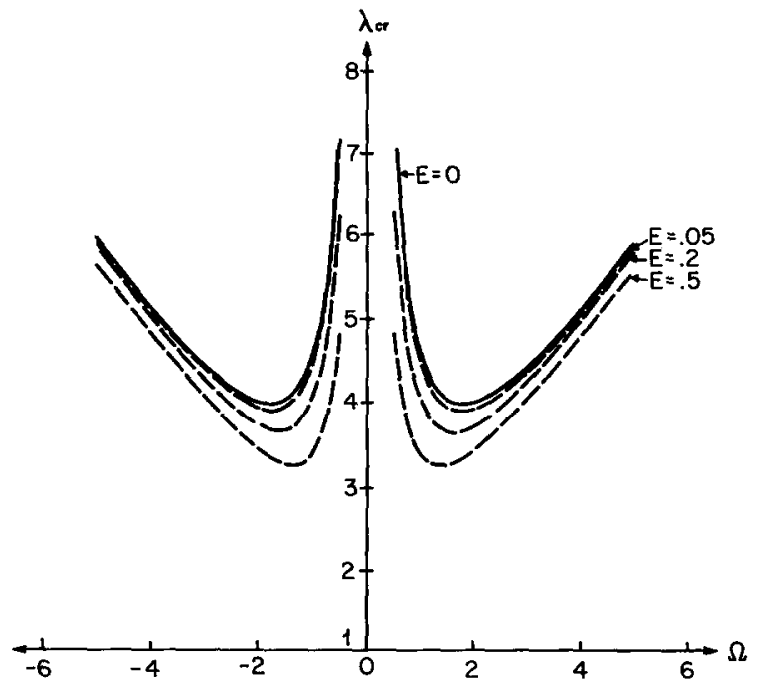

FIG. 3B. Dependence of lowest critical load $\lambda_{c r}$ on the nondimensional axial wavenumber $\Omega$ for various values of the initial thickness parameter $E$ in the case of an internally pressurized neo-Hookean tube.

the shell is due to the choice of load parameter adopted here. Had the stretch ratio of the innermost fiber been chosen instead, as the load parameter, the corresponding critical load would be an increasing function of the tube thickness as intuitively expected.

Unfortunately no direct comparison is possible between the present results in Fig. 3B and HAUGHTON and OGDEN's (1979) calculations for the internally pressurized neo-Hookean solid, in view of their lack of critical load calculations for the asymmetric 


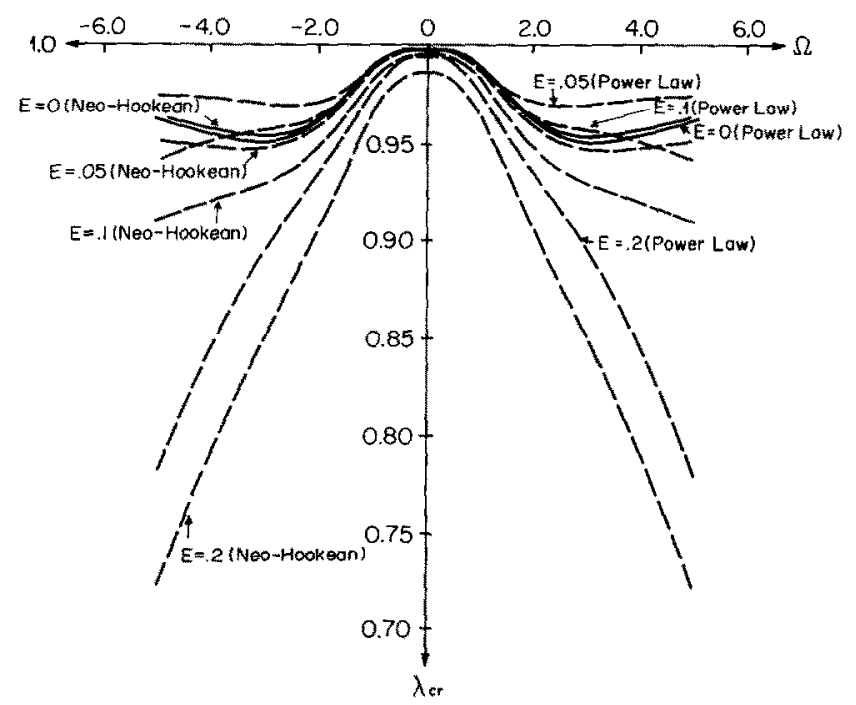

FIG. 4. Dependence of the highest critical load $\lambda_{c r}$ on the nondimensional axial wavenumber $\Omega$ for various values of the initial thickness parameter $E$ in the case of an externally pressurized power law $(m=1)$ and neo-Hookean tube.

bifurcation mode case. The apparent discrepancy in the results for the axisymmetric mode $(n=0)$ where, in contrast to the present calculations, an axisymmetric bifurcation is predicted by the aforementioned authors, is due to our restrictive boundary conditions for the admissible modes in the $n=0$ case [see (3.18)].

Similar calculations for the dependence of the critical load $\lambda_{\mathrm{er}}$ on the nondimensional axial wavenumber $\Omega$ in the case of an externally pressurized power law $(m=1)$ and neo-Hookean material, for different values of the initial thickness parameter $E$, are depicted in Fig. 4. All calculations correspond to a circumferential wavenumber $n=n_{c}=2$. Note that in agreement with our assumptions in Sections 4 and 5 the numerically calculated maximum critical load always occurs in the plane strain condition $\Omega=0$. As expected, the critical loads for the neo-Hookean material are for $E \geqslant 0.05$ lower than those corresponding to the power law one, in view of the second's softer response (i.e. lower incremental moduli) for a given strain level.

Finally the comparison between the asymptotic theory predictions and the numerical calculations for shells of various thicknesses is shown in Figs. 5-7. The comparison of the numerically calculated critical load to its three term $O\left(E^{2}\right)$ expansion of equation (4.19) in the inside pressure case or five term $O\left(E^{4}\right)$ expansion of equation (4.22) in the outside pressure casc, is depicted in Fig. 5 for the power law material. Figure 6 gives the corresponding results for the neo-Hookean material in which case the asymptotic inner pressure critical load is given by the two term expansion $O(E)$ in $(5.9)_{1}$ while the asymptotic result for the outer pressure case is still given by (4.22). Finally the numerically calculated and asymptotically approximated critical axial wavenumber $\Omega_{c r}$, using the two term $O(E)$ expansion in $(5.9)_{2}$, for the case of the neoHookean tube are compared in Fig. 7. In all the last three figures dotted lines represent finite element results while the solid ones correspond to the asymptotic approximations. Note that in all the cases examined above, discrepancies of the order 


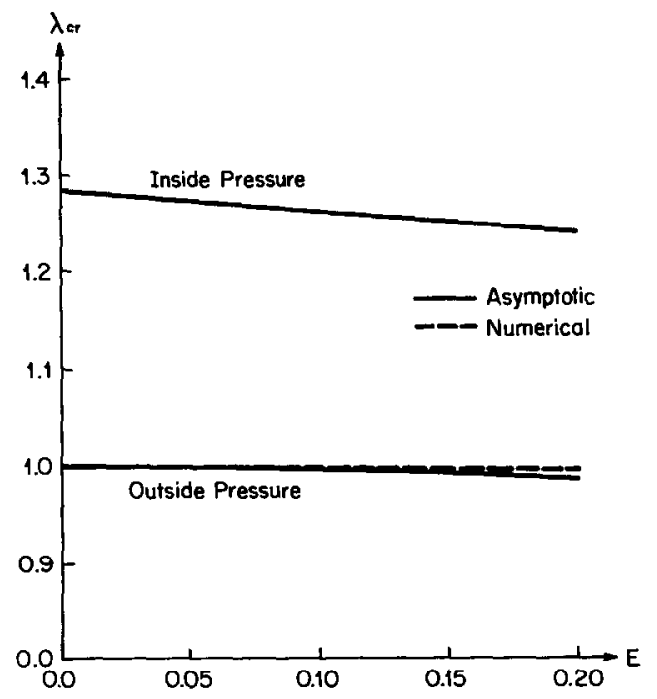

FIG. 5. Comparison between the numerically calculated (--- line) and asymptotically approximated ( - line) critical load $\lambda_{c r}$ for the internally and externally pressurized power law tube for different values of the initial thickness parameter $E$.

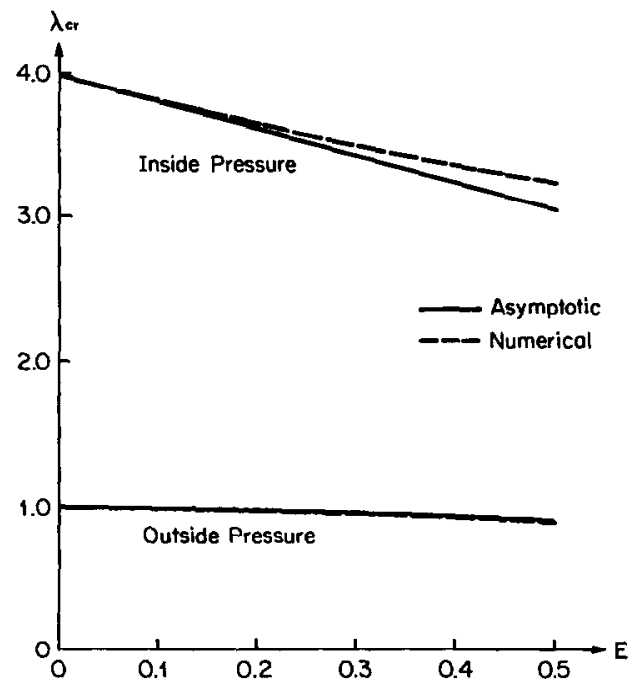

FIG. 6. Comparison between the numerically calculated (--- line) and asymptotically approximated ( - line) critical load $\lambda_{c r}$ for the internally and externally pressurized neo-Hookean tube for different values of the initial thickness parameter $E$.

of $1 \%$ or less were found for values of the initial thickness parameter $E \leqslant 0.2$ which already corresponds to quite thick shells.

\section{Concluding Remarks}

The purpose of this work is to propose a consistent and unified approach for the analysis of buckling instabilities in shell-like structures of arbitrary thickness. In place 


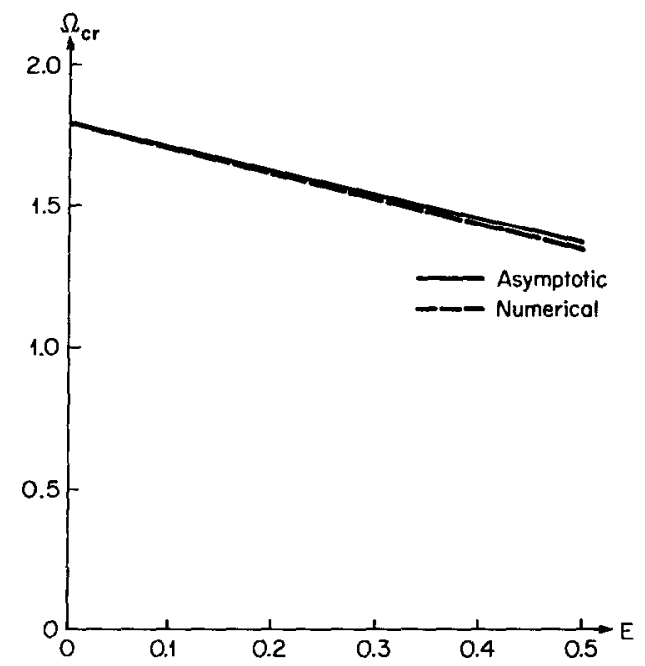

Fig. 7. Comparison between the numerically calculated $(--$ line) and asymptotically approximated ( $\longrightarrow$ critical axial wavenumber $\Omega_{c r}$ for the internally pressurized neo-Hookean tube for different values of the initial thickness parameter $E$.

of the classical approach of linearizing about the critical load the governing nonlinear shell equations, in the present approach the full three dimensional linearized bifurcation equations for the solid are asymptotically expanded with respect to its thickness.

The natural selection for the small parameter is the initial shell thickness versus some characteristic length of the undeformed middle surface. In the limiting process considered, the undeformed middle surface remains fixed as the initial thickness tends to zero, thus making the limiting process dependent only on one small parameter. As seen from the general formulation which is briefly outlined in Section 2, the method results in the solution of a sequence of two dimensional boundary value problems whose domain is the reference middle surface of the shell. In view of the significant algebraic complication inherent in the general problem formulation for the case of an arbitrary shell, the method is illustrated by means of a non-trivial example (namely the pressurization of an infinitely long thick cylindrical tube), whose asymptotic solution (unlike the full three dimensional one) can be calculated analytically. The asymptotic results obtained for the critical load and modes show very good agreement with the full scale finite element computations even for relatively thick shells. The power of the proposed method can thus be appreciated from the fact that it provides new results for a fairly well studied problem and in addition provides analytical proof for certain phenomena that have so far becn found only numerically.

Although the purpose of the example is essentially a means of illustration of the proposed method (and not of course an exhaustive study of the corresponding boundary value problem), the theoretical advantages of the proposed method can be appreciated through this example. These advantages (in comparison with the classical approach to the shell buckling problem) are essentially two : the consistency in obtaining the critical loads and modes independently of the nonlinear shell theory employed and the possiblity of obtaining higher order terms if necessary for the thickness dependence of these quantities. 
There are also some practical advantages which are expected from the proposed method, especially in sheet metal forming applications where an initially flat sheet is plastically deformed between a set of dies in order to form a final product. In view of the compressive forces generated in the deforming sheet, wrinkling instabilities are as inevitable as they are unwanted. Since the majority of numerical codes dealing with sheet forming problems are membrane ones, they cannot predict these instabilities. The classical way of dealing with the problem would be the use of a nonlinear shell theory which, in addition to being costlier, presents the obvious problem of its proper selection. The deeper in the plastic range the buckling occurs, the higher the discrepancy of results from theory to theory since the linearization of a different model is involved every time. One can show however (the proof being omitted as outside the scope of this presentation) that the quantity of practical interest, i.e. the lowest order term in the critical load expansion, depends solely on the structure's membrane solution, thus avoiding the costly (and ambiguous) nonlinear shell theory calculations by making use of the already existing membrane codes.

Of course since the issue of shell buckling is a fairly old and difficult one, the present work is only a small step in providing a better approach for this classical problem. There are numerous related issues, some more practical (there is more than one way to formulate the arbitrary mid-surface shell problem in view of the large strains involved as one can see from the problem treated here) and some more theoretical (concerning the method's convergence) which merit further investigation and which we hope to be able to address in the near future.

\section{ACKNOWLEDGEMENTS}

Support for this work from the General Motors Corporation is gratefully appreciated. One of us (N. Triantafyllidis) would also like to acknowledge helpful discussions with Dr. R. Stevenson in relation to the metal plasticity applications of this work. The same author is grateful for stimulating discussions during the earlier stages of this work to Prof. Nguyen Quoc Son of the Solid Mechanics Laboratory of the Ecole Polytechnique of Palaiseau, France, during his stay as a Distinguished Visiting Professor at the University of Michigan.

\section{REFERENCES}

BUDIANSKY, B.

ChU, C. C.

DESTUYNDER, $\mathrm{P}$.

Haughton, D. M. and OGDEN, R. W.

HILL, R.

HILL, $R$.

HILL, R.
1974 Adv. Appl. Mech. 14 (edited by C. S. Y YH), p. 1, Academic Press, New York.

1979 J. Appl. Mech. 46, 889.

1980 On the Justification of Plate and Shell Models Via Asymptotic Methods (in French), Doc. Thesis, Univ. of Paris VI.

1979 J. Mech. Phys. Solids 27, 179 and 489.

1957 J. Mech. Phys. Solids 5, 229.

1958 J. Mech. Phys. Solids 6, 236.

1969 Problems in Mechanics: Deformation of Solid Bodies, Novozilov Anniversary Volume p. 459 , Leningrad. 
HILL, R. and

HUTCHinson, J. W.

HuTCHINSON, J. W.

KOITER, W. T.

LARSON, M.

Larson, M., Needleman, A., TVERGAARD, V. and STORAKERS, B.

STORAKERS, B.

STÖREN, S. and RICE, J. R.

Timoshenko, S. P. and

GERE, J. M.
1975 J. Mech. Phys. Solids 23, 239.

1974 Adv. Appl. Mech. 14 (edited by C. S. YIH), p. 67, Academic Press, New York.

1945 On the Stability of Elastic Equilibrium (in Dutch), Doc. Thesis, Delft Univ. Amsterdam.

1978 Instabilities in Closed End Thick Walled Cylinders Subject to Internal Pressure (in Swedish), Diploma Thesis, Royal Inst. of Technology, Stockholm.

$1982 J$. Mech. Phys. Solids 30, 121.

1971 J. Mech. Phys. Solids 19, 339.

1975 J. Mech. Phys. Solids 23, 421.

1961 Theory of Elastic Stability, 2nd ed. McGraw-Hill, New York. 